\title{
Cracking and damage from crystallization in pores: Coupled chemo-hydro-mechanics and phase-field modeling
}

\author{
Jinhyun Choo $^{\mathrm{a}, \mathrm{b}, *}$, WaiChing Sun ${ }^{\mathrm{a}}$ \\ ${ }^{a}$ Department of Civil Engineering and Engineering Mechanics, Columbia University, New Work, NY 10027, USA \\ ${ }^{\mathrm{b}}$ Department of Civil Engineering, The University of Hong Kong, Pokfulam, Hong Kong
}

Received 16 August 2017; received in revised form 18 November 2017; accepted 24 January 2018

Available online 7 February 2018

\begin{abstract}
Cracking and damage from crystallization of minerals in pores center on a wide range of problems, from weathering and deterioration of structures to storage of $\mathrm{CO}_{2}$ via in situ carbonation. Here we develop a theoretical and computational framework for modeling these crystallization-induced deformation and fracture in fluid-infiltrated porous materials. Conservation laws are formulated for coupled chemo-hydro-mechanical processes in a multiphase material composed of the solid matrix, liquid solution, gas, and crystals. We then derive an expression for the effective stress tensor that is energy-conjugate to the strain rate of a porous material containing crystals growing in pores. This form of effective stress incorporates the excess pore pressure exerted by crystal growth - the crystallization pressure - which has been recognized as the direct cause of deformation and fracture during crystallization in pores. Continuum thermodynamics is further exploited to formalize a constitutive framework for porous media subject to crystal growth. The chemo-hydro-mechanical model is then coupled with a phase-field approach to fracture which enables simulation of complex fractures without explicitly tracking their geometry. For robust and efficient solution of the initial-boundary value problem at hand, we utilize a combination of finite element and finite volume methods and devise a block-partitioned preconditioning strategy. Through numerical examples we demonstrate the capability of the proposed modeling framework for simulating complex interactions among unsaturated flow, crystallization kinetics, and cracking in the solid matrix. (C) 2018 Elsevier B.V. All rights reserved.
\end{abstract}

Keywords: In-pore crystallization; Chemo-hydro-mechanics; Fracture; Phase field; Effective stress; Reactive flow

\section{Introduction}

Growth of mineral crystals in pores can give rise to severe damage and cracks in the host material. These coupled chemo-hydro-mechanical processes are now central to a number of problems in our society. A well-known example is weathering and deterioration of historic and building structures due to salt crystallization. Many of these structures are comprised of materials prone to invasion of salt water (e.g., stone), so they can be severely damaged when

\footnotetext{
* Corresponding author at: Department of Civil Engineering, The University of Hong Kong, Pokfulam, Hong Kong.

E-mail addresses: jchoo@hku.hk (J. Choo),wsun@columbia.edu (W. Sun).
} 


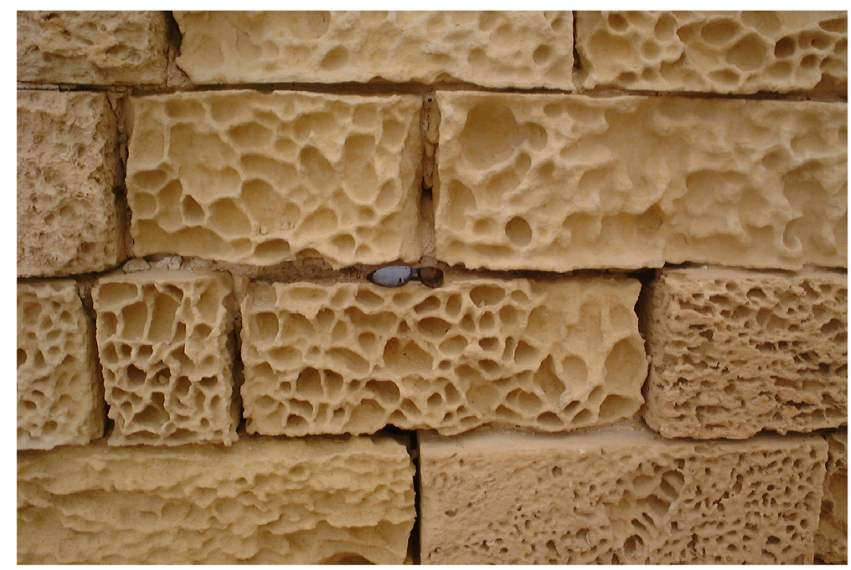

Fig. 1. Example of damage in building stones by crystallization of salts in pores. Source: Photograph by Suzanne MacLeod, distributed under a CC-BY 2.0 license.

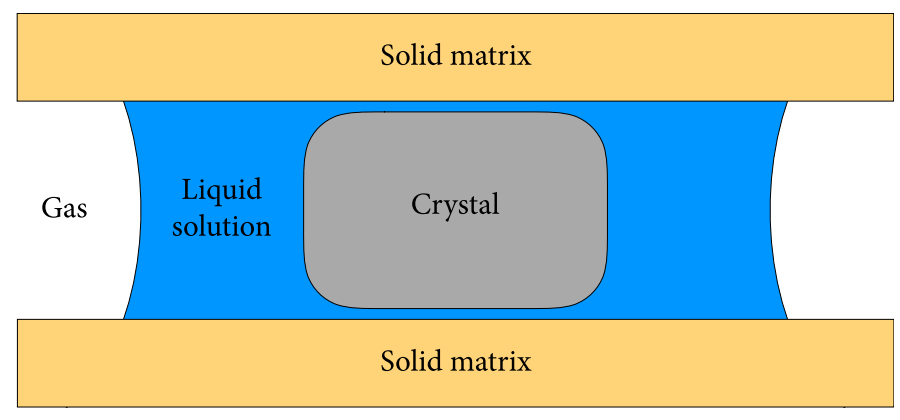

Fig. 2. Schematic illustration of a crystal inside a pore. Note that the crystal is confined within the liquid solution, not being in contact with the solid matrix. Similar illustrations have been presented in $[12,15]$.

salt minerals grow inside the pores. See Fig. 1 for example. Preventing this type of damage has been a critical element of conservation of cultural heritage and structures around the world [1-6]. Also, crystallization of minerals in the subsurface can trigger ground heaving that severely damages buildings and geotechnical structures [7-9]. Furthermore, reaction-driven cracking during mineral hydration, carbonation, and oxidation is a key consideration for deploying a promising strategy for geologic carbon storage that transforms $\mathrm{CO}_{2}$ into solid carbonate minerals [10].

Addressing the problem of cracking and damage from crystallization in pores requires us, as a first step, to understand its fundamental mechanism. Fig. 2 schematically shows how a crystal is present inside a pore space. Importantly, the crystal is usually confined within a liquid solution, maintaining a thin liquid film between its surface and the solid pore wall (see Scherer [11,12] for detailed explanations). The liquid solution surrounding the crystal allows it to continuously grow and push on the pore wall. This process of crystal growth generates an excess pressure on the solid matrix, which is commonly referred to as the crystallization pressure in a number of previous studies (e.g., [12-19]). These studies have proposed expressions of the crystallization pressure, showing that this pressure can far exceed the tensile strengths of many porous materials. This affirms that the crystallization pressure is the direct cause of fracturing and damage during crystallization in pores, and thus, an accurate prediction of the crystallization pressure per se is an important research problem. However, an expression for the crystallization pressure alone is insufficient for tackling real-world problems such as those mentioned above, because the scales of these problems are orders of magnitude larger than the scale of pores. Also necessitated is a continuum-scale modeling framework that allows us to simulate and predict how crystallization pressures would evolve in space and time and ultimately affect the problem at the field scale. The necessity of such a predictive modeling framework is the motivation of this work. 
Continuum modeling of fracturing by in-pore crystallization poses two significant challenges. First, it requires a mathematical formulation that encapsulates complex interactions among chemical reactions, fluid flow, and solid deformation in porous materials. A coupled formulation for such chemo-hydro-mechanical processes is not only difficult to develop in a theoretically consistent manner, but also challenging to solve numerically since the flow, transport, and reaction processes involve multiple length and time scales. Second, as can be seen from Fig. 1, the fracturing process of interest entails extremely complicated geometries which cannot be idealized as a set of sharp discontinuities. Obviously, algorithmic capture of such complex geometries is very unwieldy and onerous. Since these two types of challenges are interwoven herein, theoretical and computational modeling of crystallization-induced fracturing is a particularly demanding task.

Modeling frameworks that address both of these theoretical and computational challenges remain scarce. To our knowledge, Coussy [20] introduced the first chemo-hydro-mechanical framework for deformation and fracture from in-pore crystallization of minerals. His work presented significant contributions to theoretical aspects, but it remained unclear how the theory can be applied to construct an initial-boundary value problem of the crystallizationinduced fracturing process. Some more recent studies proposed computational models that paved the way to numerical simulation of the crystallization problem (e.g., [21,22]). Yet, challenges remain for both the theory and computation. First, theoretical formulations in the past studies show significant disagreement, particularly with respect to the definition of effective stress which governs the constitutive behavior of the solid matrix. Second, the existing computational models appear insufficient for addressing complex cracking and damage processes from crystallization. For example, Koniorczyk and Gawin [21] used linear elasticity without consideration of fracture. Derluyn et al. [22] employed a simple local damage model along with elasticity, but their work was limited to identification of crack nucleation by comparing the effective stress and the tensile strength in a 1-D setting. Such an approach would be inappropriate for delineation of the onset and evolution of damage and cracking zones in multi-dimensional problems. Also, their use of the standard, continuous Galerkin finite element method (FEM) may not be an optimal choice, since it could suffer from numerical stability problems arising from the advective transport of minerals. To address the stability problem, Derluyn et al. [22] smoothed equations related to crystallization kinetics. However, they also found that the smoothing parameters can plague the physics significantly. Another issue is that their work used a one-way coupled staggered scheme, which is presumably because computational cost for fully coupled chemo-hydromechanics is prohibitively expensive without a carefully designed solution strategy. All of the aforementioned aspects indicate that a significant amount of more work is necessary to advance the theory and computation of crystallizationinduced cracking and damage in porous materials.

The purpose of this work is to develop a more theoretically grounded, computationally efficient modeling framework for crystallization-induced deformation and fracture in porous materials. To this end, we first draw on recent advances in continuum modeling of coupled multiphysics in porous materials. For theoretically consistent modeling of the coupled chemo-hydro-mechanical problem at hand, we use continuum principles of thermodynamics which have been the basis of rigorous poromechanical frameworks in the literature [23-29]. The use of thermodynamic arguments provides a systematic procedure to derive physically meaningful forms of effective stress and constitutive laws for coupled multiphysical processes. Notably, the poromechanical frameworks developed in this way have demonstrated their ability to reproduce a variety of real-world observations (e.g., [29-31]). Then, for obtaining a stable numerical solution to the problem, we utilize the finite volume method (FVM) for the fluid flow and transport problem which involves advection phenomena, and the continuous Galerkin finite element method for the solid deformation problem which does not. Furthermore, we introduce a three-field block-partitioned solver that enables one to solve the coupled chemo-hydro-mechanical problem with an affordable computational cost.

As for the modeling of cracking and damage, we adopt a phase-field approach to fracture which has emerged as an efficient means for simulating complicated cracks without explicitly tracking their geometry (e.g., [32-54]). This feature of phase-field modeling is particularly desirable for our purpose since most (if not all) observed cracks due to in-pore crystallization are extremely difficult to delineate geometrically. Also, in most cases, crystallization in pores gives rise to distributed microcracks, resulting in rounded damage zones like those shown in Fig. 1. Such complex damage patterns can also be captured by a phase-field model, because it can be regarded as a particular class of nonlocal gradient damage models [55]. As such, in this work we adopt a phase-field model of fracture to simulate both distributed damages and localized cracks by in-pore crystallization, in a mesh insensitive manner. In doing so, 


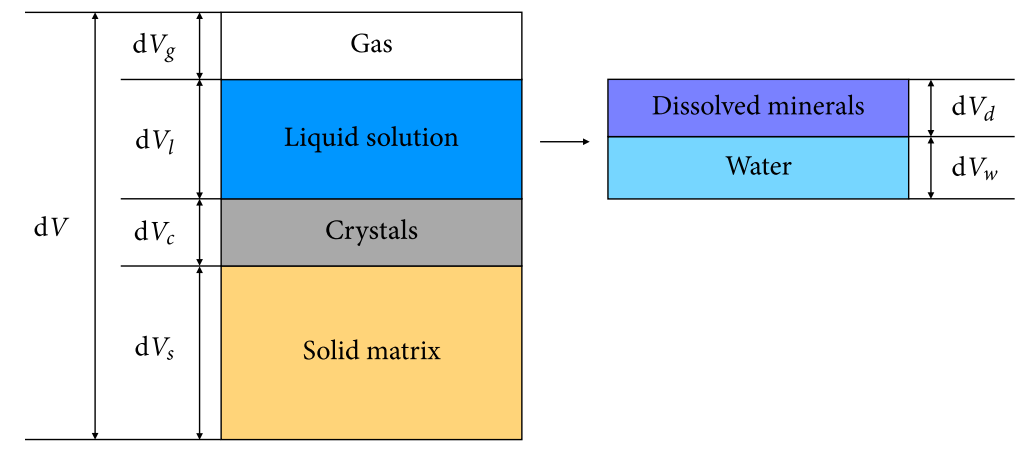

Fig. 3. Elementary volume representation of a four-phase mixture composed of the solid matrix, liquid solution, gas, and crystals. The liquid solution is also a sub-mixture of water and dissolved minerals.

we also propose a way to estimate the length regularization parameter of the phase-field model, by drawing on an empirical relationship between the fracture toughness values and the tensile strengths of geomaterials.

The paper is organized as follows. In Section 2, we formulate balance laws for a porous continuum containing liquid solution, gas, and crystals in pores. Subsequently, in Section 3 we use thermodynamic arguments in conjunction with the balance laws to derive suitable expressions for effective stress and multiphysical constitutive relations. In Section 4, we derive a phase-field formulation of brittle fracture as a balance law such that it can be augmented to the coupled chemo-hydro-mechanics formulation developed in the previous sections. We then discretize the resulting formulation in Section 5 via a combination of finite element and finite volume methods, and devise a block-partitioned iterative solver for the coupled problem. In Section 6 we present numerical examples demonstrating the validity and performance of the proposed modeling framework for simulating complex chemo-hydro-mechanical and fracturing processes from crystallization of minerals in pores.

\section{Conservation laws}

In this section, we develop conservation laws for coupled chemo-hydro-mechanical processes in mineralcontaining, partially saturated porous materials. We first introduce a continuum mixture representation of this type of porous material, and then formulate balance laws for its mass, linear momentum, and energy. At this point, we note that the purpose of this and the next section is to formulate a general mathematical model amenable to being combined with various methods for modeling cracking and damage. Later in Section 4, we will adopt a specific method, namely the phase-field method.

\subsection{Continuum representation}

Using mixture theory we conceptualize the material of interest as a multiphase continuum in which the solid matrix, liquid solution, gas, and mineral crystals are overlapped. See Fig. 3 for an elementary volume representation of this four-phase mixture. We define the volume fractions of the constituent phases as

$$
\phi^{s}:=\frac{\mathrm{d} V_{s}}{\mathrm{~d} V}, \quad \phi^{l}:=\frac{\mathrm{d} V_{l}}{\mathrm{~d} V}, \quad \phi^{g}:=\frac{\mathrm{d} V_{g}}{\mathrm{~d} V}, \quad \phi^{c}:=\frac{\mathrm{d} V_{c}}{\mathrm{~d} V}, \quad \phi^{s}+\sum_{\alpha=l, g, c} \phi^{\alpha}=1,
$$

where the index $s$ refers to the solid matrix, $l$ the liquid solution, $g$ the gas, and $c$ the crystals. It is noted that an index is used as a subscript when referring to an intrinsic property of a constituent phase, whereas it is used as a superscript when referring to a partial property of the mixture. We define the saturation ratios of the phases in the pore space the liquid solution, the gas, and the crystals - as

$$
S^{l}:=\frac{\phi^{l}}{1-\phi^{s}}, \quad S^{g}:=\frac{\phi^{g}}{1-\phi^{s}}, \quad S^{c}:=\frac{\phi^{c}}{1-\phi^{s}}, \quad S^{l}+S^{g}+S^{c}=1 .
$$


Let $\rho_{s}, \rho_{l}$, and $\rho_{c}$ denote the intrinsic mass densities of the solid, the liquid solution, and the mineral crystals, respectively. The partial mass densities of the constituent phases are given by

$$
\rho^{s}:=\phi^{s} \rho_{s}, \quad \rho^{l}:=\phi^{l} \rho_{l}, \quad \rho^{g}:=\phi^{g} \rho_{g}, \quad \rho^{c}:=\phi^{c} \rho_{c}, \quad \rho^{s}+\sum_{\alpha=l, g, c} \rho^{\alpha}=\rho,
$$

where $\rho$ is the mass density of the entire mixture.

As shown in Fig. 3, the liquid solution itself is also a mixture composed of water and dissolved minerals. Let the index $w$ denote the water and $d$ denote the dissolved minerals. Their global volume fractions are given by

$$
\phi^{w}:=\frac{\mathrm{d} V_{w}}{\mathrm{~d} V}, \quad \phi^{d}:=\frac{\mathrm{d} V_{d}}{\mathrm{~d} V}, \quad \phi^{w}+\phi^{d}=\phi^{l},
$$

and their saturation ratios are

$$
S^{w}:=\frac{\phi^{w}}{1-\phi^{s}}, \quad S^{d}:=\frac{\phi^{d}}{1-\phi^{s}}, \quad S^{w}+S^{d}=S^{l} .
$$

We also define local volume fractions of the water and dissolved minerals as

$$
\psi^{w}:=\phi^{w} / \phi^{l}=S^{w} / S^{l}, \quad \psi^{d}:=\phi^{d} / \phi^{l}=S^{d} / S^{l}, \quad \psi^{w}+\psi^{d}=1 .
$$

Similarly, their partial densities are defined as

$$
\rho^{w}:=\phi^{w} \rho_{w}=\phi^{l} \psi^{w} \rho_{w}, \quad \rho^{d}:=\phi^{d} \rho_{d}=\phi^{l} \psi^{d} \rho_{d}, \quad \rho^{w}+\rho^{d}=\rho^{l} .
$$

Note that the intrinsic density of dissolved minerals $\rho_{d}$ is identical to $\rho_{c}$, but we have used $\rho_{d}$ for notational purposes. Using the above variables, we can write the mass fraction of minerals dissolved in the solution as

$$
c:=\frac{\rho^{d}}{\rho^{l}}=\frac{\rho^{d}}{\rho^{w}+\rho^{d}}=\frac{\psi^{d} \rho_{d}}{\psi^{w} \rho_{w}+\psi^{d} \rho_{d}} .
$$

\subsection{Balance of mass}

To derive conservation laws for this mixture, we use a kinematic description that traces the motion of the solid matrix. Let an overdot denote the material time derivative with respect to the motion of the solid matrix. Then we can write balance equations for the masses of the solid, the liquid solution, the gas, the dissolved minerals, and the mineral crystals as

$$
\begin{aligned}
& \dot{\rho}^{s}+\rho^{s} \nabla \cdot \boldsymbol{v}=m_{s}, \\
& \dot{\rho}^{l}+\rho^{l} \nabla \cdot \boldsymbol{v}+\nabla \cdot \boldsymbol{w}_{l}=m_{l}, \\
& \dot{\rho}^{g}+\rho^{g} \nabla \cdot \boldsymbol{v}+\nabla \cdot \boldsymbol{w}_{g}=m_{g}, \\
& \dot{\rho}^{d}+\rho^{d} \nabla \cdot \boldsymbol{v}+\nabla \cdot \boldsymbol{w}_{d}=m_{d}, \\
& \dot{\rho}^{c}+\rho^{c} \nabla \cdot \boldsymbol{v}+\nabla \cdot \boldsymbol{w}_{c}=m_{c},
\end{aligned}
$$

respectively. Here, $\boldsymbol{v}$ is the velocity of the solid matrix, $m_{\alpha}$ is the rate of mass production for phase $\alpha$, and $\boldsymbol{w}_{\alpha}$ is the Eulerian relative mass flow vector of phase $\alpha$ with respect to the solid matrix, which is given by

$$
\boldsymbol{w}_{\alpha}:=\rho^{\alpha} \tilde{\boldsymbol{v}}_{\alpha}, \quad \tilde{\boldsymbol{v}}_{\alpha}:=\boldsymbol{v}_{\alpha}-\boldsymbol{v}, \quad \alpha=l, g, d, c,
$$

with $\boldsymbol{v}_{\alpha}$ being defined as the velocity of phase $\alpha$. For the dissolved minerals in the liquid solution, the relative mass flow is decomposed into convective and diffusive/dispersive parts as

$$
\boldsymbol{w}_{d}=c \boldsymbol{w}_{l}+\boldsymbol{j},
$$

where $\boldsymbol{j}$ is the diffusive/dispersive mass flux of the minerals in the solution.

At this point, we introduce a few assumptions that simplify mathematical expressions in the succeeding development. First, we assume that the water and crystal phases are incompressible and the solid matrix undergoes infinitesimal deformations. Second, adopting the assumption in the fluid flow model of Castellazzi et al. [56], we postulate that only the dissolved and crystallized minerals exchange masses among the five constituent phases, i.e., $m_{s}=m_{g}=0$ and $m_{l}=m_{d}=-m_{c}$. Third, we consider that momentum and pressure of the water and dissolved minerals in the liquid solution are under equilibrium. 


\subsection{Balance of linear momentum}

Balance of linear momentum for the solid, liquid solution, gas, and crystals, respectively, can be written as

$$
\begin{aligned}
& \nabla \cdot \boldsymbol{\sigma}^{s}+\rho^{s} \boldsymbol{g}+\boldsymbol{h}^{s}=\rho^{s} \boldsymbol{a}, \\
& \nabla \cdot \boldsymbol{\sigma}^{l}+\rho^{l} \boldsymbol{g}+\boldsymbol{h}^{l}=\rho^{l} \boldsymbol{a}_{l}-m_{l} \boldsymbol{v}_{l}, \\
& \nabla \cdot \boldsymbol{\sigma}^{g}+\rho^{g} \boldsymbol{g}+\boldsymbol{h}^{g}=\rho^{g} \boldsymbol{a}_{g}, \\
& \nabla \cdot \boldsymbol{\sigma}^{c}+\rho^{c} \boldsymbol{g}+\boldsymbol{h}^{c}=\rho^{c} \boldsymbol{a}_{c}+m_{c} \boldsymbol{v}_{c},
\end{aligned}
$$

where $\boldsymbol{\sigma}^{\alpha}$ is the partial (Cauchy) stress tensor of phase $\alpha, \boldsymbol{a}$ and $\boldsymbol{a}_{\alpha}$ are the acceleration of the solid matrix and phase $\alpha$, respectively, and $\boldsymbol{h}^{\alpha}$ is the frictional drag force vector on phase $\alpha$, which is subject to the constraint

$$
\boldsymbol{h}^{s}+\boldsymbol{h}^{l}+\boldsymbol{h}^{g}+\boldsymbol{h}^{c}=\mathbf{0} .
$$

Summing up the above equations gives an expression for balance of linear momentum for the entire mixture, which reads

$$
\nabla \cdot \boldsymbol{\sigma}+\rho \boldsymbol{g}=\rho \boldsymbol{a}+\sum_{\alpha=l, g, c} \rho^{\alpha} \tilde{\boldsymbol{a}}_{\alpha}+m_{c}\left(\tilde{\boldsymbol{v}}_{l}+\tilde{\boldsymbol{v}}_{c}\right),
$$

where $\tilde{\boldsymbol{a}}_{\alpha}:=\boldsymbol{a}_{\alpha}-\boldsymbol{a}$. Here, $\boldsymbol{\sigma}:=\boldsymbol{\sigma}^{s}+\boldsymbol{\sigma}^{l}+\boldsymbol{\sigma}^{g}+\boldsymbol{\sigma}^{c}$ is the total stress tensor in the mixture.

\subsection{Balance of energy}

Let $P$ denote the total power, $K$ the kinetic energy, and $I$ the internal energy in the mixture. Then balance of energy for the mixture can be expressed as

$$
P=\dot{K}+\dot{I} .
$$

The rate of change of kinetic energy in an arbitrary volume $\mathcal{V}$ of the mixture is given by

$$
\dot{K}=\int_{\mathcal{V}} \rho^{s} \boldsymbol{a} \cdot \boldsymbol{v} \mathrm{d} V+\sum_{\alpha=l, g, c} \int_{\mathcal{V}} \rho^{\alpha} \boldsymbol{a}_{\alpha} \cdot \boldsymbol{v}_{\alpha} \mathrm{d} V+\sum_{\alpha=l, g, c} \int_{\mathcal{V}} \frac{1}{2} m_{\alpha} \boldsymbol{v}_{\alpha} \cdot \boldsymbol{v}_{\alpha} \mathrm{d} V,
$$

and the rate of change of internal energy is

$$
\dot{I}=\int_{\mathcal{V}} \rho \dot{e} \mathrm{~d} V
$$

where $\dot{e}$ is the rate of change of internal energy per unit total mass of the mixture.

The total power is the sum of the mechanical power $P^{m}$ and the non-mechanical power $P^{n}$, i.e., $P=P^{m}+P^{n}$. In this work, we consider isothermal conditions in which $P^{n}=0$. Let us introduce the infinitesimal strain tensor for the solid $\boldsymbol{\varepsilon}=\nabla^{\mathrm{s}} \boldsymbol{u}=\left(\nabla \boldsymbol{u}+\nabla^{\mathrm{T}} \boldsymbol{u}\right) / 2$ where $\boldsymbol{u}$ is the solid displacement vector. Similarly we introduce infinitesimal strain tensors $\boldsymbol{\varepsilon}_{\alpha}$ for phase $\alpha$. The mechanical power is then given by

$$
\begin{aligned}
P^{m}= & \int_{\mathcal{V}}\left(\boldsymbol{\sigma}^{s}: \dot{\boldsymbol{\varepsilon}}+\sum_{\alpha=l, g, c} \boldsymbol{\sigma}^{\alpha}: \dot{\boldsymbol{\varepsilon}}_{\alpha}\right) \mathrm{d} V \\
& +\int_{\mathcal{V}}\left(\nabla \cdot \boldsymbol{\sigma}^{s} \cdot \boldsymbol{v}+\boldsymbol{h}^{s} \cdot \boldsymbol{v}+\rho^{s} \boldsymbol{g} \cdot \boldsymbol{v}\right) \mathrm{d} V \\
& +\sum_{\alpha=l, g, c} \int_{\mathcal{V}}\left(\nabla \cdot \boldsymbol{\sigma}^{\alpha} \cdot \boldsymbol{v}_{\alpha}+\boldsymbol{h}^{\alpha} \cdot \boldsymbol{v}_{\alpha}+\rho^{\alpha} \boldsymbol{g} \cdot \boldsymbol{v}_{\alpha}\right) \mathrm{d} V .
\end{aligned}
$$

We now substitute above expressions into $\dot{I}=P-\dot{K}$, impose the balance of linear momentum for each phase, and localize the resulting integral. Then, we can express the rate of change of internal energy as

$$
\rho \dot{e}=\sigma^{s}: \dot{\boldsymbol{\varepsilon}}+\sum_{\alpha=l, g, c} \boldsymbol{\sigma}^{\alpha}: \dot{\boldsymbol{\varepsilon}}_{\alpha}-\sum_{\alpha=l, g, c} \frac{1}{2} m_{\alpha} \boldsymbol{v}_{\alpha} \cdot \boldsymbol{v}_{\alpha} .
$$


This energy balance equation will be the starting point of the next section where we derive suitable forms of the effective stress tensor and constitutive laws.

\section{Effective stress and constitutive framework}

The purpose of this section is to identify physically meaningful constitutive relations that close the formulation. For this purpose, we begin by deriving a suitable form of the effective stress tensor, which is a necessary step for constitutive modeling of a deformable porous material infiltrated by fluid. We do this derivation based on a thermodynamic argument that the effective stress should be energy-conjugate to the strain rate tensor. From the effective stress derivation we identify several groups of energy-conjugate pairs, and we use them as a guide to develop constitutive relations for coupled chemo-hydro-mechanical modeling.

\subsection{Effective stress}

Our goal here is to find the form of stress measure energy-conjugate to the strain rate tensor for the solid matrix, which, by definition, corresponds to the effective stress in a fluid-infiltrated porous material. For this purpose we apply the procedure developed by Borja [24], which was originally proposed for porous media without any crystals, to materials containing solid crystals in their pores. In doing so, we postulate that the phases in the pores - the liquid solution, gas, and crystals - can only carry volumetric stresses. This postulate is reasonable for most pore fluids of interests. For the crystals, the postulate may be justified by the fact that they are usually floating in the liquid solution, as depicted in Fig. 2 previously. See the same reasoning in $\mathrm{Na}$ and Sun [57] for incorporating ice crystals into effective stress in frozen soils. Then, we can express the partial stress tensors of the in-pore phases as

$$
\boldsymbol{\sigma}^{\alpha}=-\phi^{\alpha} p_{\alpha} \mathbf{1}, \quad \alpha=l, g, c,
$$

where $p_{\alpha}$ is the intrinsic pressure of phase $\alpha$ and $\mathbf{1}$ is the second-order identity tensor. Substituting the above expression into Eq. (26) gives

$$
\rho \dot{\boldsymbol{e}}=\sigma: \dot{\boldsymbol{\varepsilon}}-\sum_{\alpha=l, g, c} \phi^{\alpha}\left(\nabla \cdot \tilde{\boldsymbol{v}}_{\alpha}\right) p_{\alpha}-\sum_{\alpha=l, g, c} \frac{1}{2} m_{\alpha} \boldsymbol{v}_{\alpha} \cdot \boldsymbol{v}_{\alpha} .
$$

From Eq. (28), we can see that the second term on the right hand side is directly related to the solid deformation (because $\nabla \cdot \tilde{\boldsymbol{v}}_{\alpha}=\nabla \cdot \boldsymbol{v}_{\alpha}-\nabla \cdot \boldsymbol{v}$ ), whereas the last term is irrelevant to the solid deformation. Thus we now seek to extract the contribution of $\phi^{\alpha}\left(\nabla \cdot \tilde{\boldsymbol{v}}_{\alpha}\right)$ to the solid deformation. The mass balance equations for the liquid solution, gas, and crystals can be rewritten as

$$
\dot{\phi}^{\alpha}+\phi^{\alpha} \nabla \cdot \boldsymbol{v}+\phi^{\alpha} \nabla \cdot \tilde{\boldsymbol{v}}_{\alpha}+\nabla \phi^{\alpha} \cdot \tilde{\boldsymbol{v}}_{\alpha}=\frac{m_{\alpha}}{\rho_{\alpha}}, \quad \alpha=l, g, c,
$$

and rearranging the above equations gives

$$
\phi^{\alpha} \nabla \cdot \tilde{\boldsymbol{v}}_{\alpha}=-\phi^{\alpha} \nabla \cdot \boldsymbol{v}-\dot{\phi}^{\alpha}-\nabla \phi^{\alpha} \cdot \tilde{\boldsymbol{v}}_{\alpha}+\frac{m_{\alpha}}{\rho_{\alpha}}, \quad \alpha=l, g, c .
$$

Now we look for an alternative expression for $\dot{\phi}^{\alpha}$ as it is also related to the solid deformation. Recalling that $\phi^{\alpha}=\left(1-\phi^{s}\right) S^{\alpha}$ for $\alpha=l, g, c$, the material time derivative of $\phi^{\alpha}$ is given by

$$
\dot{\phi}^{\alpha}=\left(1-\phi^{s}\right) \dot{S}^{\alpha}-S^{\alpha} \dot{\phi}^{s}, \quad \alpha=l, g, c .
$$

The remaining challenge is to have an explicit expression for the last term, $\dot{\phi}^{s}$. Borja [24] has shown that, in the case of barotropic (isothermal) flow for the solid, the mass balance equation for the solid can be expressed as

$$
\dot{\phi}^{s}+\left(\phi^{s}-b\right) \nabla \cdot \boldsymbol{v}=0,
$$

where $b:=K / K_{s}$, with $K$ and $K_{s}$ being the bulk moduli of the solid matrix and the solid constituent, respectively. Inserting Eq. (32) into Eq. (31), we get

$$
\dot{\phi}^{\alpha}=\left(1-\phi^{s}\right) \dot{S}^{\alpha}+S^{\alpha}\left(\phi^{s}-b\right) \nabla \cdot v, \quad \alpha=l, g, c .
$$


Using the above equations, we can rewrite Eq. (30) as

$$
\phi^{\alpha} \nabla \cdot \tilde{\boldsymbol{v}}_{\alpha}=-\left[\phi^{\alpha}+S^{\alpha}\left(\phi^{s}-b\right)\right] \nabla \cdot \boldsymbol{v}-\left(1-\phi^{s}\right) \dot{S}^{\alpha}-\nabla \phi^{\alpha} \cdot \tilde{\boldsymbol{v}}_{\alpha}+\frac{m_{\alpha}}{\rho_{\alpha}}, \quad \alpha=l, g, c .
$$

Here, the coefficients of $\nabla \cdot v$ can be simplified to

$$
\phi^{\alpha}+S^{\alpha}\left(\phi^{s}-b\right)=\left(1-\phi^{s}\right) S^{\alpha}+S^{\alpha}\left(\phi^{s}-b\right)=(1-b) S^{\alpha}=B S^{\alpha}, \quad \alpha=l, g, c,
$$

where $B:=1-b=1-K / K_{s}$ is the well-known Biot coefficient. Finally, we can decompose $\phi^{\alpha} \nabla \cdot \tilde{\boldsymbol{v}}_{\alpha}$ in Eq. (28) as

$$
\phi^{\alpha} \nabla \cdot \tilde{\boldsymbol{v}}_{\alpha}=-B S^{\alpha} \nabla \cdot \boldsymbol{v}-\left(1-\phi^{s}\right) \dot{S}^{\alpha}-\nabla \phi^{\alpha} \cdot \tilde{\boldsymbol{v}}_{\alpha}+\frac{m_{\alpha}}{\rho_{\alpha}}, \quad \alpha=l, g, c .
$$

Observe that the first term on the right hand side contains $\nabla \cdot v$, which is the rate of volume change of the solid matrix.

Substituting Eq. (36) into Eq. (28), we can rewrite the energy balance equation as

$$
\begin{aligned}
\rho \dot{\boldsymbol{e}}= & \sigma^{\prime}: \dot{\boldsymbol{\varepsilon}}+\sum_{\alpha=l, g, c}\left(1-\phi^{s}\right) \dot{S}^{\alpha} p_{\alpha}+\sum_{\alpha=l, g, c}\left(\nabla \phi^{\alpha} \cdot \tilde{\boldsymbol{v}}_{\alpha}\right) p_{\alpha} \\
& +\sum_{\alpha=l, g, c}\left(\frac{m_{\alpha}}{\rho_{\alpha}}\right) p_{\alpha}-\sum_{\alpha=l, g, c} \frac{1}{2} m_{\alpha} \boldsymbol{v}_{\alpha} \cdot \boldsymbol{v}_{\alpha},
\end{aligned}
$$

where

$$
\boldsymbol{\sigma}^{\prime}=\boldsymbol{\sigma}+B \bar{p} \mathbf{1}=\boldsymbol{\sigma}+B \sum_{\alpha=l, g, c}\left(S^{\alpha} p_{\alpha}\right) \mathbf{1}
$$

is the stress measure energy-conjugate to the strain rate tensor, which, by definition, is the effective stress. The "pore pressure" in this form of effective stress, denoted by $\bar{p}$, is

$$
\bar{p}:=\sum_{\alpha=l, g, c}\left(S^{\alpha} p_{\alpha}\right)=S^{l} p_{l}+S^{g} p_{g}+S^{c} p_{c}=\left(S^{l}+S^{c}\right) p_{l}+S^{g} p_{g}+S^{c}\left(p_{c}-p_{l}\right),
$$

which is the mean of the liquid, gas, and crystal pressures weighted by their volume fractions (saturation ratios).

In the absence of crystals (i.e., $S^{c}=0$ ), Eq. (38) boils down to the thermodynamically consistent effective stress tensor in unsaturated porous media derived by Borja [24], which specializes to the well-known Terzaghi's effective stress [58] in the limit of full saturation and $B=1$. However, when a crystal is growing inside a liquid solution in confined pore space, the crystal growth exerts significant excess pressure on the solution film between the crystal and surrounding solid particles. This pressure - which is commonly referred to as the crystallization pressure in the literature - can make $p_{c}$ far greater than $p_{l}$. Then the effective stress could become tensile even as the total stress is compressive, resulting in damage and fracture. Our derivation thus formally shows that the crystallization pressure can be a direct driver of deformation and fracture from crystallization in pores. Given its significance, the crystallization pressure is hereafter denoted by $p_{\mathrm{cr}}$, i.e.,

$$
p_{\mathrm{cr}}:=p_{c}-p_{l}
$$

\subsection{Constitutive framework}

Having derived the form of effective stress, we further exploit the energy-conjugate pairs in the energy balance equation to identify suitable forms of constitutive laws. We particularly extend the procedure of Borja and coworkers [26,27], which has been used for unsaturated porous materials without crystals, to materials containing crystals in pores. This procedure simply interprets the type and form of constitutive relationship that each energyconjugate pair suggests, without performing a thorough thermodynamic analysis. The reason is that the standard argument will eventually require variables in each energy-conjugate pair to be linked via a constitutive relation, for ensuring the second law of thermodynamics. Therefore, in what follows, we interpret the implication of each energy-conjugate pair, and adopt one of the widely used constitutive models in the literature. The selected constitutive model may not agree with the most general form allowed by a thermodynamic analysis. Note, however, that the specific model is just chosen for constructing a particular class of the general framework we propose in this work-it can readily be replaced by another constitutive model depending on the purpose of modeling. Also, for brevity, the 
following discussion focuses on new aspects emerging from the presence of the crystals. Detailed explanations of the other aspects unrelated to the crystals can be found in [23-27].

For a clearer interpretation, we first simplify some terms in Eq. (37). Since $S^{l}+S^{g}+S^{c}=1$, we have $\dot{S}^{l}=-\dot{S}^{g}-\dot{S}^{c}$. Thus the second term in Eq. (37) can be expressed as

$$
\sum_{\alpha=l, g, c}\left(1-\phi^{s}\right) \dot{S}^{\alpha} p_{\alpha}=\left(1-\phi^{s}\right) \dot{S}^{c} p_{\mathrm{cr}}+\left(1-\phi^{s}\right) \dot{S}^{g} p_{\mathrm{ca}}
$$

where the capillary pressure (suction) is defined in the last term as $p_{\mathrm{ca}}:=p_{g}-p_{l}$. Also, the third and fourth terms in Eq. (37) can be combined as

$$
\sum_{\alpha=l, g, c} \frac{m_{\alpha}}{\rho_{\alpha}} p_{\alpha}-\sum_{\alpha=l, g, c} \frac{1}{2} m_{\alpha} \boldsymbol{v}_{\alpha} \cdot \boldsymbol{v}_{\alpha}=\sum_{\alpha=l, g, c} m_{\alpha}\left[\frac{p_{\alpha}}{\rho_{\alpha}}-\frac{1}{2} \tilde{\boldsymbol{v}}_{\alpha} \cdot \tilde{\boldsymbol{v}}_{\alpha}\right] .
$$

Therefore, under isothermal conditions, we can rewrite the energy balance equation as

$$
\begin{aligned}
\rho \dot{e}= & \sigma^{\prime}: \dot{\boldsymbol{\varepsilon}}+\left(1-\phi^{s}\right) \dot{S}^{c} p_{\mathrm{cr}}+\left(1-\phi^{s}\right) \dot{S}^{g} p_{\mathrm{ca}} \\
& +\sum_{\alpha=l, g, c}\left(\nabla \phi^{\alpha} \cdot \tilde{\boldsymbol{v}}_{\alpha}\right) p_{\alpha}+\sum_{\alpha=l, g, c} m_{\alpha}\left[\frac{p_{\alpha}}{\rho_{\alpha}}-\frac{1}{2} \tilde{\boldsymbol{v}}_{\alpha} \cdot \tilde{\boldsymbol{v}}_{\alpha}\right] .
\end{aligned}
$$

From the above equation we identify five groups of energy-conjugate pairs, which means that five types of constitutive relations are necessary to ensure non-negative entropy production. For each group, we can introduce a constitutive relation as follows.

The first energy-conjugate pair, from which we have defined the effective stress, is the mechanical power produced by the deformation of the solid matrix. For this pair, it is natural to introduce a stress-strain relation of the form

$$
\dot{\boldsymbol{\sigma}}^{\prime}=\mathbb{C}: \dot{\boldsymbol{\varepsilon}},
$$

where $\mathbb{C}$ is a fourth-order tangent stiffness tensor. In this work, we shall assume that the solid behavior is linear elastic. This assumption may not allow us to fully describe the complex stiffness of geomaterials [59-61], but it is often good enough for modeling brittle fracture on which we focus in this work.

The second pair contains the crystallization pressure and the rate of volume change of the crystals. Obviously this pair implies a constitutive law for the crystallization pressure. Notably, the derived energy-conjugate relationship is consistent with an equation for crystallization pressure put forward by Kelemen and Hirth [18], which can be expressed as

$$
p_{\mathrm{cr}}=-\frac{\Delta \psi}{\Delta V_{s}},
$$

where $\Delta \psi$ is the change in Helmholtz free energy and $\Delta V_{s}$ is the difference in volume between the solid products and the solid reactants. A number of specific expressions have been proposed for the crystallization pressure (e.g., [12-19]). Here we use the classical Correns' equation [13,14], given by

$$
p_{\mathrm{cr}}=\frac{R T}{V_{m}} \ln \left(\frac{c}{c_{\mathrm{eq}}}\right) \text {, }
$$

where $R$ is the gas constant, $T$ is the temperature in Kelvin, $V_{m}$ is the molar volume of the mineral, and $c_{\text {eq }}$ is the equilibrium mass fraction at which the liquid is saturated by the mineral. This equation has later been shown to overlook the role of chemical activities in the crystallization process [15,62], but it gives reasonable predictions compared with experimental data of salt crystallization.

The third pair contains the gas saturation ratio and the capillary pressure. Given that the gas saturation is determined by the liquid saturation, these variables may be related by a water retention law for unsaturated geomaterials. A common choice is the van Genuchten equation [63], given by

$$
S^{l}\left(p_{\mathrm{ca}}\right)=S_{1}^{l}+\left(S_{2}^{l}-S_{1}^{l}\right)\left[1+\left(p_{\mathrm{ca}} / \alpha_{\mathrm{ca}}\right)^{n}\right]^{-m} .
$$

This equation requires four material parameters: the residual liquid saturation $S_{1}^{l} \geq 0$, the maximum liquid saturation $S_{2}^{l} \leq 1$, the scaling capillary pressure $\alpha_{\mathrm{ca}}$, and the exponent $n$. Another exponent $m$ is related to $n$ via $m=1-1 / n$. This equation is originally developed for geomaterials infiltrated by freshwater, so it may be unable to accommodate 
some important aspects emerging from crystal growth in pores. However, the effects of crystallization on the retention behavior have only become a subject of research recently (e.g., [64]), and they are still far from being encapsulated into a water retention equation. For this reason, the original van Genuchten equation is used herein, but it can be modified easily when the effects of in-pore crystals become quantified. On a related note, Derluyn et al. [22] also used the van Genuchten model and their simulation results showed good agreements with experimental data even when salt minerals were crystallized. Also noted is that a very recent phase-field model employing the van Genuchten equation has successfully reproduced qualitative patterns of drying-induced cracks, see Cajuhi et al. [53].

The variables contained in the fourth group of energy-conjugate pairs are the relative velocity, pressure, and volume fractions of all phases inside the pores. This means that we need constitutive laws for flows of the in-pore phases relative to the solid matrix. For the liquid solution, we use the multiphase extension of Darcy's law, which can be written as

$$
\boldsymbol{q}^{l}:=-\frac{k_{r} \boldsymbol{k}}{\mu_{l}} \cdot\left(\nabla p_{l}-\rho_{l} \boldsymbol{g}\right),
$$

where $\boldsymbol{q}^{l}:=\phi^{l} \tilde{\boldsymbol{v}}_{l}$ is the seepage velocity, $\mu_{l}$ is the dynamic viscosity of the liquid solution, $k_{r}$ is the relative permeability, and $\boldsymbol{k}$ is the second-order absolute permeability tensor. We assume that the permeability tensor of the intact solid matrix is isotropic, i.e., $\boldsymbol{k}=k \mathbf{1}$, and will discuss its anisotropic evolution by fracturing in the next section. Usually $k$ is assumed to be constant when the solid deformation is infinitesimal. In our problem, however, crystals can clog a significant portion of the pore space. To accommodate this clogging effect, here we consider $k$ a function of pore volume, adopting the Kozeny-Carman equation. The equation can be written in a normalized form

$$
k=k_{0}\left(\frac{\left(1-\phi_{0}\right)^{2}}{\phi_{0}^{3}}\right)\left(\frac{\phi^{3}}{(1-\phi)^{2}}\right),
$$

where $\phi:=1-\phi^{s}$ is the porosity, and $k_{0}$ and $\phi_{0}$ are the reference values of the absolute permeability and the porosity, respectively. The relative permeability is regarded as a function of saturation. When the water retention behavior is modeled by the van Genuchten equation, the relative permeability can be expressed as

$$
k_{r}=\theta^{1 / 2}\left[1-\left(1-\theta^{1 / m}\right)^{m}\right]^{2}, \quad \theta=\frac{S^{l}-S_{1}^{l}}{S_{2}^{l}-S_{1}^{l}} .
$$

The relative flow of the dissolved mineral is comprised of convective and diffusive/dispersive parts, as in Eq. (15). The convective part is described by Darcy's law for the liquid solution presented above. For the diffusive/dispersive part, we introduce a linear diffusion equation

$$
\boldsymbol{j}=-\rho_{l} D \nabla c,
$$

where $D$ is the diffusion coefficient for the dissolved minerals. For this coefficient we adopt an equation used in Derluyn et al. [22], which takes the form of $D=\left(D_{m} / \tau\right)\left(1-\phi^{s}\right)\left(S^{l}\right)^{1.6}$ with $D_{\tau}$ being the molecular diffusivity of the mineral and $\tau$ being the tortuosity of the porous media. In what follows, we shall assume that the gas pressure is atmospheric (i.e, $p_{g} \approx 0$ ) and that the relative velocity of the crystal is negligibly small (i.e., $\tilde{\boldsymbol{v}}_{c} \approx 0$ ). These assumptions, which have been introduced to other poromechanical models as well (e.g., [27,57]), allow us to neglect constitutive laws for the relative flows of the gas and crystal phases.

Lastly, the fifth group of energy-conjugate pairs contains the rate of mass exchange, intrinsic density, pressure, and relative velocity of each phase inside the pores. Because $m_{c}=-m_{l}$ and $m_{g}=0$, we only need to consider a constitutive law for $m_{c}$. It is noted that the velocity has already been related to the pressure, and the pressure related to the saturation. This means that a constitutive law that relates the mass exchange, density, and that saturation terms can satisfy this energy-conjugacy. In fact, by definition, such a constitutive law corresponds to a kinetic equation for crystal growth and dissolution. Here we adopt the kinetic equation proposed by Espinosa-Marzal et al. [65], which has later become the common choice of computational models of salt crystallization (e.g., $[21,22,56])$. This equation can be expressed as

$$
m_{c}=\left\{\begin{array}{cl}
\left(1-\phi^{s}\right) S^{l} K_{c}\left(U-U_{\mathrm{thr}}\right)^{g_{c}} & \text { if } U \geq U_{\mathrm{thr}}, \\
-\left(1-\phi^{s}\right) S^{l} K_{c}(1.0-U)^{g_{c}} & \text { if } U<1 \text { and } S^{c}>0,
\end{array}\right.
$$

where $K_{c}>0$ and $g_{c}>0$ are kinetic parameters, $U \geq 0$ is the supersaturation ratio, and $U_{\mathrm{thr}} \geq 1$ is the threshold value of $U$ for crystal growth which depends on the type of mineral. Usually $U_{\text {thr }} \geq 1$ for primary crystallization but 
$U_{\text {thr }}=1$ once the crystallization process has begun. Multiple definitions are possible for the supersaturation ratio $U$, and here we consider $U=c / c_{0}$ for consistency with Eq. (46). Note that the first equation in Eq. (52) represents a crystal growth process $\left(m_{c} \geq 0\right.$ if $U \geq U_{\text {thr }}$ ), whereas the second equation represents a crystal dissolution process ( $m_{c}<0$ if $U<1$ and $S^{c}>0$ ). Note, however, that complex changes in the material's internal structure by dissolution (e.g., the formation of a sensitive clay by leaching) are beyond the modeling capacities of this formulation employing linear elasticity.

So far, we have developed a general modeling framework for coupled chemo-hydro-mechanical processes in fluid-infiltrated porous materials containing dissolved and crystallized minerals. In doing so, we have constructed a particular class of the framework by selecting a set of constitutive laws. It is again noted that our selection is just a specific choice, and other sets of constitutive laws would work equally well. Similarly, while we adopt a phase-field approach to fracture in the following, other approaches for similar purposes are also compatible with our development herein.

\section{Phase-field formulation for fracture}

In this section, we present a phase-field model of fracture driven by the effective stress derived in the previous section. To be consistent with the foregoing continuum mechanics approach, here we derive the phase-field model as a balance law of microforces. Microforce balance derivations of phase-field models of fractures have been presented in several previous studies [37,41,42,46,54]. Among them, we adopt the derivation procedure of Choo and Sun [54], which differs from other microforce derivations because it views crack growth as a thermodynamically irreversible process. The motivation and implication of this derivation are explained in detail in Choo and Sun [54].

Without loss of generality, in this section we consider a "dry" porous solid in which the liquid, gas, and crystal phases are absent. This simplification is just to prevent a proliferation of terms unrelated to the fracturing process. Again, an important premise in continuum poromechanics is that all mechanical processes - including the fracturing process which we will describe as the evolution of the phase-field variable - are driven by the effective stress. This means that terms independent of the effective stress will not affect the formulation that follow. Thus, for brevity, the terms unrelated to the deformation and fracture of the solid matrix are omitted in this section.

\subsection{Phase-field approximation of fracture surfaces}

We first introduce a phase-field approximation of fracture geometry, which, in essence, is an approximation of a sharp discontinuity as a diffuse interface. Let $\Gamma$ denote a set of discontinuous fractures inside the body $\Omega$. The total area of the fracture surfaces is given by

$$
A_{\Gamma}:=\int_{\Gamma} \mathrm{d} A,
$$

which is an area integral over $\Gamma$. Calculating this integral during the evolution of fracture is an infeasible task because tracing the change in $\Gamma$ is extremely difficult in most cases. To circumvent this difficulty, we seek to transform an area integral over the evolving domain $\Gamma$ into a volume integral over the fixed domain $\Omega$. For this purpose we define a phase-field variable $d \in[0,1]$, which denotes an intact state by $d=0$ and a fully cracked state by $d=1$. Naturally $d$ can be understood as a damage variable. Using this phase-field variable, we introduce a crack density functional $\Gamma_{\mathrm{d}}(d, \nabla d)$ such that

$$
A_{\Gamma} \approx A_{\Gamma_{\mathrm{d}}}:=\int_{\Omega} \Gamma_{\mathrm{d}}(d, \nabla d) \mathrm{d} V,
$$

where the last integral is defined over the volume $\Omega$. In this work, we adopt a widely used crack density functional of the form

$$
\Gamma_{\mathrm{d}}(d, \nabla d)=\frac{d^{2}}{2 l}+\frac{l}{2}|\nabla d|^{2},
$$

where $l>0$ is the length parameter for the phase-field regularization. The smaller the length parameter $l$, the closer the phase-field approximation to the original sharp discontinuity. 


\subsection{Balance law derivation of phase-field evolution}

To derive a governing equation for the phase-field variable, we make use of the microforce approach developed by Gurtin [66], which has proven useful to derive phase-field models [37,41,42,46,54] and other types of models [67,68] within the framework of continuum mechanics. The first step of this approach is to postulate the existence of a microforce system in which the phase-field variable $d$ (referred to as the order parameter in [66]) is energy-conjugate to an internal microforce $\pi$ and a surface microforce $\zeta$. Consider an arbitrary volume $\mathcal{V}$ with boundary $\partial \mathcal{V}$ in this microforce system. The balance of microforce over the volume $\mathcal{V}$ is given by

$$
\int_{\partial \mathcal{V}} \zeta \mathrm{d} A+\int_{\mathcal{V}} \pi \mathrm{d} V=0
$$

Denoting the unit normal vector of $\partial \mathcal{V}$ by $\boldsymbol{n}$, we also introduce a microforce traction vector $\boldsymbol{\xi}$ such that $\zeta=\boldsymbol{\xi} \cdot \boldsymbol{n}$. Then, by applying the divergence theorem and noting the arbitrariness of $\mathcal{V}$, we obtain a localized form of Eq. (56) as

$$
\nabla \cdot \xi+\pi=0 .
$$

The internal and surface microforces, which are energy-conjugate to the phase-field variable $d$, should evolve such that this balance law is satisfied. Therefore this microforce balance law serves as a governing equation for the evolution of phase field—equivalently, the damage and fracturing process. In the following, we derive specific forms of $\pi$ and $\xi$ based on thermodynamic arguments.

The mechanical power in the microforce system is given by

$$
\tilde{P}^{m}=\int_{\partial \mathcal{V}}(\xi \cdot \boldsymbol{n}) \dot{d} \mathrm{~d} A=\int_{\mathcal{V}}(\xi \cdot \nabla \dot{d}-\pi \dot{d}) \mathrm{d} V
$$

Incorporating this additional mechanical power as well as keeping the effective stress power only, we can rewrite the balance of energy as

$$
\rho \dot{e}=\sigma^{\prime}: \dot{\boldsymbol{\varepsilon}}+\xi \cdot \nabla \dot{d}-\pi \dot{d} .
$$

We will exploit the second law of thermodynamics to derive expressions for the effective stress tensor and the microforce variables. To this end, we first consider a stored energy density function of the form

$$
\psi(\boldsymbol{\varepsilon}, d)=g(d) W(\boldsymbol{\varepsilon}) .
$$

Here, $W(\varepsilon)$ denotes the strain energy stored in the undamaged material, and $g(d) \in[0,1]$ is the so-called degradation function which should satisfy $g(0)=1$ and $g(1)=0$. For now we consider general forms of $W(\varepsilon)$ and $g(d)$, and will discuss their specific forms later in this section.

At this point, it is noted that the energy used to create a fracture surface does not enter the stored energy function because crack growth is considered fully dissipative a priori herein. This is consistent with the stored energy functions used in variational frameworks for fracture (e.g., $[34,47])$, but different from those assumed in other balance law derivations of phase-field models based on microforce arguments $[37,41,42,46]$. More specifically, the stored energy functions in previous balance law derivations contain the fracture energy, so their derivations lead to a thermodynamic implication that crack growth is a reversible process in a rate-independent setting. This implication would be appropriate for clean fracture surfaces that can heal under highly controlled conditions. However, here we prefer to view crack growth as an irreversible process, since crack healing phenomena in geomaterials are usually inconsistent with the thermodynamic definition of a reversible process. See Choo and Sun [54] for a more detailed discussion on this aspect.

Having defined the stored energy density, we can write the dissipation inequality as

$$
\mathcal{D}=\sigma^{\prime}: \dot{\varepsilon}+\xi \cdot \nabla \dot{d}-\pi \dot{d}-\dot{\psi} \geq 0 .
$$

The time derivative of the stored energy function is given by

$$
\dot{\psi}(\boldsymbol{\varepsilon}, d)=\frac{\partial \psi}{\partial \boldsymbol{\varepsilon}}: \dot{\boldsymbol{\varepsilon}}+\frac{\partial \psi}{\partial d} \dot{d} .
$$

Substituting Eq. (62) into Eq. (61), we get an alternative expression for the dissipation inequality as

$$
\mathcal{D}=\left(\boldsymbol{\sigma}^{\prime}-\frac{\partial \psi}{\partial \boldsymbol{\varepsilon}}\right): \dot{\boldsymbol{\varepsilon}}-\left(\pi-\frac{\partial \psi}{\partial d}\right) \dot{d}+\boldsymbol{\xi} \cdot \nabla \dot{d} \geq 0 .
$$


To ensure non-negative dissipation of the stress power irrespective of $\dot{\varepsilon}$, the effective stress should be related to the stored energy. This standard argument leads to a hyperelastic relation of the form

$$
\boldsymbol{\sigma}^{\prime}=\frac{\partial \psi}{\partial \boldsymbol{\varepsilon}}
$$

Next, as done in $[67,68]$ for deriving other types of models, we assume that the internal microforce is additively decomposed into two parts, the energetic (non-dissipative) part $\pi^{\text {en }}$ and the dissipative part $\pi^{\text {dis }}$. In other words, $\pi=\pi^{\text {en }}+\pi^{\text {dis }}$. The same argument that we used to get Eq. (64) yields the following expression for the energetic part:

$$
\pi^{\mathrm{en}}=-\frac{\partial \psi}{\partial d} .
$$

Inserting Eqs. (64) and (65) into Eq. (63) gives the reduced dissipation inequality of the form

$$
\mathcal{D}^{\mathrm{f}}=\xi \cdot \nabla \dot{d}-\pi^{\mathrm{dis}} \dot{d} \geq 0 .
$$

Because the material is considered elastic, the dissipation is solely attributed to the evolution of the phase-field variable $d$, or crack growth.

To arrive at specific expressions for $\xi$ and $\pi^{\text {dis }}$, we now postulate that cracks are created in a way that they maximize the energy dissipation. This postulate is consistent with the Griffith theory of brittle fracture [69], and has been central to several variational frameworks for phase-field fracture (e.g., $[34,47])$. Our task is then to find expressions for the microforce variables that maximize $\mathcal{D}^{\mathrm{f}}$ defined in Eq. (66). Equivalently, we seek to minimize the negative of the reduced dissipation functional, given by

$$
-\mathcal{D}^{\mathrm{f}}=-\xi \cdot \nabla \dot{d}+\pi^{\mathrm{dis}} \dot{d} \leq 0 .
$$

The arguments of this functional, $\dot{d}$ and $\nabla \dot{d}$, are indeed subject to a constraint in that these variables should form a phase-field approximation of fracture. The specific expression for this constraint can be obtained by taking time derivative of the crack density functional, Eq. (55). This procedure gives

$$
\dot{\Gamma}_{\mathrm{d}}(d, \nabla d)=\left(\frac{d}{l}\right) \dot{d}+l \nabla d(\nabla \dot{d}) .
$$

In the cases we consider, $\dot{\Gamma}_{\mathrm{d}}(d, \nabla d) \geq 0$ (crack irreversibility) and $\dot{\Gamma}_{\mathrm{d}}(d, \nabla d)<\infty$ (finite speed of crack propagation). Given this constraint, we can write a Lagrangian for this constrained minimization problem as

$$
\mathcal{L}(\dot{d}, \nabla \dot{d}, \lambda):=-\xi \cdot \nabla \dot{d}+\pi^{\mathrm{dis}} \dot{d}+\lambda\left[\left(\frac{d}{l}\right) \dot{d}+l \nabla d(\nabla \dot{d})-\dot{\Gamma}_{\mathrm{d}}\right],
$$

where $\lambda$ is the Lagrange multiplier of this problem. Invoking the stationary condition of this Lagrangian gives expressions for $\boldsymbol{\xi}$ and $\pi^{\text {dis }}$ as follows:

$$
\begin{aligned}
& \delta_{\nabla \dot{d}} \mathcal{L}=-\xi+\lambda l \nabla d=0 \quad \rightarrow \quad \xi=\lambda l \nabla d, \\
& \delta_{\dot{d}} \mathcal{L}=\pi^{\mathrm{dis}}+\lambda\left(\frac{d}{l}\right)=0 \quad \rightarrow \quad \pi^{\mathrm{dis}}=-\lambda\left(\frac{d}{l}\right) .
\end{aligned}
$$

The remaining task is to identify the meaning of the Lagrange multiplier, $\lambda$, in the context of our problem. To this end, we substitute the results of Eqs. (70) and (71) into $\mathcal{D}^{\mathrm{f}}$ in Eq. (66), which is the energy dissipation per unit volume. Integrating the resulting dissipation density over the domain $\Omega$ with the crack surface $\Gamma_{\mathrm{d}}$ gives

$$
\int_{\Omega} \mathcal{D}^{\mathrm{f}} \mathrm{d} V=\int_{\Omega} \lambda\left[\left(\frac{d}{l}\right) \dot{d}+l \nabla d(\nabla \dot{d})\right] \mathrm{d} V=\int_{\Omega} \lambda \dot{\Gamma}_{\mathrm{d}} \mathrm{d} V \approx \frac{\mathrm{d}}{\mathrm{d} t} \int_{\Gamma_{\mathrm{d}}} \lambda \mathrm{d} A \geq 0,
$$

where the last approximation is attributed to the phase-field regularization of discontinuous surfaces. Eq. (72) shows that the Lagrange multiplier $\lambda$ can be interpreted as the energy dissipated by the creation of unit crack surface area. By definition, this energy corresponds to the critical fracture energy in fracture mechanics.

Let $\mathcal{G}_{c}$ denote the critical fracture energy. Now we can express the internal microforce as

$$
\pi=\pi^{\mathrm{en}}+\pi^{\mathrm{dis}}, \quad \pi^{\mathrm{en}}=-\frac{\partial \psi}{\partial d}=-g^{\prime}(d) W(\varepsilon), \quad \pi^{\mathrm{dis}}=-\mathcal{G}_{c}\left(\frac{d}{l}\right),
$$


and the microforce traction vector as

$$
\xi=\mathcal{G}_{c} l \nabla d .
$$

Substituting these expressions into Eq. (57), we can rewrite the governing equation for the phase-field variable as

$$
-g^{\prime}(d) W(\varepsilon)-\mathcal{G}_{c}\left(\frac{d}{l}-l \nabla \cdot(\nabla d)\right)=0 .
$$

Notably, this equation is the same as the governing equation for a phase-field model of brittle, rate-independent fracture obtained by variational and other balance law approaches, see [34,35,37,42] for example.

\subsection{Stored energy function}

Now we consider specific expressions for the stored energy function, $\psi(\varepsilon, d)=g(d) W(\varepsilon)$. The stored energy function is central to the phase-field model of fracture since it determines the effective stress tensor $\boldsymbol{\sigma}^{\prime}$, as in Eq. (64), and the energetic force driving the evolution of the phase-field variable $d$, as in Eq. (73).

First, as for the degradation function $g(d)$, we adopt the form most widely used by the phase-field modeling community, given by

$$
g(d)=(1-d)^{2} .
$$

It is noted that other forms of degradation functions have also been suggested, e.g., the cubic degradation proposed by [41].

Next, we need to determine a suitable form of $W(\varepsilon)$, the strain energy function decoupled from the phase-field variable. In doing so, we should take into account that for physically realistic results, the strain energy associated with pure compression should not give rise to fracturing. For this reason, previous studies have proposed to decompose the stored energy function as $W(\varepsilon)=W_{+}(\varepsilon)+W_{-}(\varepsilon)$, where $W_{+}(\varepsilon)$ is the fracturing part related to the tensile strain energy, and $W_{-}(\varepsilon)$ is the non-fracturing part related to the compressive strain energy. This decomposition has been mainly done via either of the following two schemes: one that uses the sign of principal strains proposed by Miehe et al. [34], and another that uses a volumetric-deviatoric split of the strain energy function proposed by Amor et al. [33]. Here we use the former one, and decompose the stored energy function of a linear elastic material as

$$
W_{+}(\boldsymbol{\varepsilon})=\frac{1}{2} \bar{\lambda}\langle\operatorname{tr}(\boldsymbol{\varepsilon})\rangle_{+}^{2}+\bar{\mu} \sum_{a=1}^{3}\left\langle\varepsilon_{a}\right\rangle_{+}^{2}, \quad W_{-}(\boldsymbol{\varepsilon})=\frac{1}{2} \bar{\lambda}\langle\operatorname{tr}(\boldsymbol{\varepsilon})\rangle_{-}^{2}+\bar{\mu} \sum_{a=1}^{3}\left\langle\varepsilon_{a}\right\rangle_{-}^{2},
$$

where $\langle\cdot\rangle_{ \pm}=(\cdot \pm|\cdot|) / 2, \bar{\lambda}$ and $\bar{\mu}$ are the Lamé parameters, and $\varepsilon_{a}$ are the principal strains.

After decomposing the strain energy as above, we apply the degradation function to the fracturing part only, i.e.,

$$
\psi(\boldsymbol{\varepsilon}, d)=g(d) W_{+}(\boldsymbol{\varepsilon})+W_{-}(\boldsymbol{\varepsilon}) .
$$

Then the effective stress is expressed as

$$
\boldsymbol{\sigma}^{\prime}=\frac{\partial \psi(\boldsymbol{\varepsilon}, d)}{\partial \boldsymbol{\varepsilon}}=g(d) \frac{\partial W_{+}(\boldsymbol{\varepsilon})}{\partial \boldsymbol{\varepsilon}}+\frac{\partial W_{-}(\boldsymbol{\varepsilon})}{\partial \boldsymbol{\varepsilon}} .
$$

Likewise, the energetic microforce is expressed as

$$
\pi_{\mathrm{en}}=-\frac{\partial \psi(\boldsymbol{\varepsilon}, d)}{\partial d}=-g^{\prime}(d) W_{+}(\boldsymbol{\varepsilon})
$$

Note that $\pi_{\text {en }}$ does not take the non-fracturing part of the strain energy, $W_{-}(\varepsilon)$.

\subsection{Thermodynamic restriction and crack irreversibility}

Our derivation leads to an expression for the dissipation inequality which needs to be satisfied for thermodynamic consistency. Rewriting Eq. (66), we can express the rate of energy dissipation in a unit volume as

$$
\mathcal{D}^{\mathrm{f}}=\mathcal{G}_{c} \dot{\Gamma}_{\mathrm{d}} \geq 0 \text {. }
$$


Because $\mathcal{G}_{c}>0$ by definition, this is equivalent to

$$
\dot{\Gamma}_{\mathrm{d}} \geq 0 \text {, }
$$

which means that crack growth should be irreversible, as we postulated in the beginning of this derivation. Integrating this equation over the entire domain leads to an expression that is identical to the crack irreversibility condition presented in variational frameworks for phase-field fracture (e.g., Eq. (20) of Miehe et al. [34]). Thus it can be concluded that our balance law derivation is consistent with the variational derivation with respect to the thermodynamic implication as well as the governing equation. Eq. (82) can also be written as

$$
\left(\frac{d}{l}\right) \dot{d}+l|\nabla d||\nabla \dot{d}| \geq 0
$$

where we have used an alternative form of the time derivative of the nonlocal term in the crack density functional. The above equation shows that the crack irreversibility condition boils down to $\dot{d} \geq 0$.

To enforce this crack irreversibility condition, $\dot{d} \geq 0$, we adopt the approach proposed by Miehe et al. [35]. The approach is to make the energetic force driving the phase-field evolution, which is denoted by $\pi^{\text {en }}$ in our derivation, a non-decreasing function even as $W_{+}(\varepsilon)$ is decreasing. Following this idea, we introduce a strain energy history functional $\mathcal{H} \geq 0$ subject to the Karush-Kuhn-Tucker condition of

$$
W_{+}-\mathcal{H} \leq 0, \quad \dot{\mathcal{H}} \geq 0, \quad \dot{\mathcal{H}}\left(W_{+}-\mathcal{H}\right)=0 .
$$

Simply speaking, $\mathcal{H}$ is the maximum of the fracturing part of the strain energy during the course of loading. Replacing the stored energy term in Eq. (75) with $\mathcal{H}$ gives a modified phase-field equation of the form

$$
-g^{\prime}(d) \mathcal{H}-\mathcal{G}_{c}\left(\frac{d}{l}-l \nabla \cdot(\nabla d)\right)=0 .
$$

Eq. (85) will be used as the governing equation for the phase-field model in the sequel.

\subsection{Permeability evolution by phase-field fracture}

Phase-field modeling of fracture in fluid-infiltrated porous media should take into account the impact of the fracturing process on fluid flow. Several types of approaches have been proposed for this purpose but with different emphases (e.g., [39,40,43,45,48,49,51]). Most of them are concerned with hydraulic fracturing whereby the rate of mass transfer between the fracture and matrix systems (leak-off) is often far lower than the fluid injection rate. The problem at hand, however, involves a quite different situation in that here crystal growth in matrix pores slowly drives fractures and significant fluid flow is expected between the fracture and matrix systems. In addition, we face a new problem that growing crystals increasingly clog the fracture aperture. Thus, an approach that views the fracture and matrix pores as a whole - rather than distinguished domains described by separate governing equations - would be more appropriate for our purpose.

Given these considerations, here we take an approach that augments an anisotropic permeability tensor describing Poiseuille flow to the absolute permeability tensor in Eq. (48). Such an approach has been advocated by Miehe and Mauthe [40,49] and Wang and Sun [70], among others. We now write the absolute permeability tensor as

$$
\boldsymbol{k}=\boldsymbol{k}_{\text {matrix }}+d^{2} \boldsymbol{k}_{\text {frac }} .
$$

Here, $\boldsymbol{k}_{\text {matrix }}$ is the isotropic permeability tensor of the solid matrix which we have discussed in the previous section. On the other hand, $\boldsymbol{k}_{\mathrm{frac}}$ is anisotropic, and given by

$$
\boldsymbol{k}_{\text {frac }}=\frac{w^{2}}{12}\left(\mathbf{1}-\boldsymbol{n}_{d} \otimes \boldsymbol{n}_{d}\right),
$$

where $\boldsymbol{n}_{d}:=\nabla d /|\nabla d|$ is the unit normal vector perpendicular to the fracture direction, and $w$ is the hydraulic aperture. Note that the hydraulic aperture herein should be a function of the crystal fraction as well as the fracture width. For simplicity, we assume that the aperture can be given by $\left(1-S^{c}\right) \bar{w}$, where $\bar{w}$ is the hydraulic aperture in the absence of crystals. For $\bar{w}$, we adopt an equation proposed by Miehe and Mauthe [40], specializing it to infinitesimal deformation conditions. The resulting form of the hydraulic aperture $w$ is given by

$$
w=\left(1-S_{c}\right) l_{\perp}\left(\boldsymbol{n}_{d} \cdot \boldsymbol{\varepsilon} \cdot \boldsymbol{n}_{d}\right),
$$


where $l_{\perp}$ is the characteristic length of a line element perpendicular to the fracture. For simplicity, we assign $l_{\perp}$ to be the mesh size $h$ as in Miehe and Mauthe [40].

\subsection{Estimation of phase-field modeling parameters for geomaterials}

Lastly, we describe how the phase-field modeling parameters are estimated in this work. A standard phase-field model of fracture requires two parameters: (1) the critical fracture energy $\mathcal{G}_{c}$, and (2) the length parameter $l$. While the two parameters have different origins - the former is from fracture mechanics theory and the latter is from the phase-field approximation of fracture - they together determine the fracturing response of a material [33,36,41,71]. In particular, the peak stress under tension, which is usually reckoned as the tensile strength, is controlled by the fracture energy, length parameter, and elasticity moduli. It is thus of significant importance to assign a suitable combination of the phase-field modeling parameters. Among them, however, the length parameter is uneasy to estimate because it is not a physically measurable quantity.

Here we devise a way to estimate the length parameter for geomaterials, drawing on an empirical relationship between the tensile strengths and the fracture toughness values of geomaterials. Let $\sigma_{t}$ denote the tensile strength of a geomaterial and $K_{I c}$ denote its mode I fracture toughness. Experimental studies have found that, for many geomaterials under quasi-static conditions, the two properties are well correlated as a linear function [72-74], given by

$$
K_{I c}=\beta \sigma_{t},
$$

where $\beta$ is the linear regression coefficient having a unit of square root meter. Example values of $\beta$ are 0.1453 for dozens of soft to hard rocks [73] and 0.3546 for compacted clays tested by Wang et al. [74]. This relationship usually shows a coefficient of determination, $r^{2}$, around 0.9 . At the same time, the tensile strength of a phase-field model is determined by the two parameters, $\mathcal{G}_{c}$ and $l$, and Young's modulus, $E$. When the degradation function is $g(d)=(1-d)^{2}$, the tensile strength is given by $[33,36]$

$$
\sigma_{t}=\frac{9}{16} \sqrt{\frac{E \mathcal{G}_{c}}{3 l}} .
$$

Also, $\mathcal{G}_{c}$ and $K_{I c}$ in linear elastic materials are related as

$$
\mathcal{G}_{c}=\frac{K_{I C}^{2}}{E^{\prime}},
$$

where $E^{\prime}:=E$ in plane stress conditions and $E^{\prime}:=E /\left(1-v^{2}\right)$ in plane strain conditions. Substituting Eqs. (90) and (91) into Eq. (89) and solving for $l$ yields

$$
l=\frac{27}{256}\left(\frac{E}{E^{\prime}}\right) \beta^{2} .
$$

Here, the unit of $l$ is meter because $\beta$ is given by a square root meter. This equation allows us to estimate $l$ from experimental data of $\sigma_{t}$ and $K_{I c}$. Once we have estimated $l$ in this way and known the tensile strength and elasticity parameters, we can find the corresponding value of $\mathcal{G}_{c}$ from Eq. (90).

Interestingly, Eq. (92) suggests that the length parameter is related to the relationship between the tensile strength and the mode I fracture toughness, rather than the values of these properties. Given that the linear relationship (89) has been found from many types of geomaterials (though not necessary valid for all types of geomaterials, see [75]), Eq. (92) may serve as a useful guide to set a length parameter for phase-field modeling of fracture in geomechanical problems.

\section{Discrete formulation}

This section develops a discrete formulation for numerical solution of the chemo-hydro-mechanics and phasefield model described so far. We begin by stating the governing equations and the relevant initial-boundary value problem. For robust and accurate spatial discretization of the coupled problem, we use the finite element method for the deformation and fracture problem (momentum balance and phase-field equations), and the finite volume method for the flow and transport problem (mass balance equations). The resulting discrete system is solved by a staggered 
scheme that sequentially updates the chemo-hydro-mechanics problem and the phase-field problem. To facilitate monolithic solution of the coupled chemo-hydro-mechanics problem, we design a block-partitioned preconditioner which significantly speeds up iterative linear solvers.

\subsection{Governing equations}

The coupled problem at hand is furnished by five governing equations that follow. The first one is the balance equation for linear momentum in the mixture. For simplicity we shall neglect the effects of inertial forces and crystallization kinetics on the linear momentum. Substituting the expression for effective stress in Eq. (38) into Eq. (21), we express the balance of linear momentum

$$
\nabla \cdot\left(\boldsymbol{\sigma}^{\prime}-B \bar{p} \mathbf{1}\right)+\rho \boldsymbol{g}=0 .
$$

The second governing equation is the mass balance for the liquid solution, which was originally given by Eq. (10). Since $\rho^{l}:=\left(1-\phi^{s}\right) S^{l} \rho_{l}$, we can expand this equation as

$$
\left(1-\phi^{s}\right)\left(\rho_{l} \dot{S}^{l}+S^{l} \dot{\rho}_{l}\right)-S^{l} \rho_{l}\left(\dot{\phi}^{s}+\phi^{s} \nabla \cdot v\right)+S^{l} \rho_{l} \nabla \cdot v+\nabla \cdot \boldsymbol{w}_{l}=m_{l} .
$$

Here, the second product can be simplified using the Biot coefficient $B$. To wit, we can rewrite Eq. (33) as

$$
\dot{\phi}^{s}+\phi^{s} \nabla \cdot \boldsymbol{v}=(1-B) \nabla \cdot \boldsymbol{v},
$$

and insert this equation into Eq. (94). Then the liquid mass balance equation takes the form

$$
\left(1-\phi^{s}\right)\left(\rho_{l} \dot{S}^{l}+S^{l} \dot{\rho}_{l}\right)+S^{l} \rho_{l} B \nabla \cdot \boldsymbol{v}+\nabla \cdot \boldsymbol{w}_{l}=m_{l} .
$$

In case $\dot{\rho}_{l}=0$ and $m_{l}=0$, the above equation degenerates to the mass balance equation of unsaturated poromechanics for shallow water problems $[24,76]$.

Third, we consider the mass balance equation for the dissolved minerals. Because $\rho^{d}=c \rho^{l}$, the mass balance equation can be rewritten as

$$
\rho^{l} \dot{c}+c\left(\dot{\rho}^{l}+\rho^{l} \nabla \cdot \boldsymbol{v}\right)+\nabla \cdot\left(c \boldsymbol{w}_{l}\right)+\nabla \cdot \boldsymbol{j}=m_{d} .
$$

In the above we have shown that

$$
\dot{\rho}^{l}+\rho^{l} \nabla \cdot \boldsymbol{v}=\left(1-\phi^{s}\right)\left(\rho_{l} \dot{S}^{l}+S^{l} \dot{\rho}_{l}\right)+S^{l} \rho_{l} B \nabla \cdot \boldsymbol{v} .
$$

Substituting the above equation and $m_{d}=m_{l}$ into Eq. (97) gives

$$
\rho^{l} \dot{c}+c\left(1-\phi^{s}\right)\left(\rho_{l} \dot{S}^{l}+S^{l} \dot{\rho}_{l}\right)+c S^{l} \rho_{l} B \nabla \cdot v+\nabla \cdot\left(c \boldsymbol{w}_{l}\right)+\nabla \cdot \boldsymbol{j}=m_{l},
$$

which is a transient advection-diffusion equation for the mass fraction, $c$.

The fourth governing equation is the mass balance for the crystals in pores. Given that $\rho^{c}=\left(1-\phi^{s}\right) S^{c} \rho_{c}$ and $\boldsymbol{w}_{c}$ has been assumed to be zero, we can rewrite Eq. (13) as

$$
\left(1-\phi^{s}\right) \rho_{c} \dot{S}^{c}-S^{c} \rho_{c}\left(\dot{\phi}^{s}+\phi^{s} \nabla \cdot v\right)+S^{c} \rho_{c} \nabla \cdot v=m_{c},
$$

Again, by appealing to Eq. (95), we get

$$
\left(1-\phi^{s}\right) \rho_{c} \dot{S}^{c}+S^{c} \rho_{c} B \nabla \cdot \boldsymbol{v}=m_{c} .
$$

Last, specifying $g^{\prime}(d)=-2(1-d)$, we rewrite the phase-field governing equation as

$$
2(1-d) \mathcal{H}-\mathcal{G}_{c}\left(\frac{d}{l}-l \nabla \cdot(\nabla d)\right)=0 .
$$

As for the primary variables of these governing equations, we select the following five fields: the displacement vector of the solid matrix $\boldsymbol{u}$, the pore pressure of the liquid solution $p:=p_{l}$, the mass fraction of the dissolved minerals $c$, the saturation ratio of the crystals $s:=S^{c}$, and the phase-field variable $d$.

We complete the statement of the problem by prescribing boundary and initial conditions as follows. Let $\Omega$ denote the domain of interest and $\partial \Omega$ denote its boundary. The boundary is suitably decomposed as: displacement and traction boundaries, $\partial \Omega_{u}$ and $\partial \Omega_{t}$; pressure and liquid flux boundaries, $\partial \Omega_{p}$ and $\partial \Omega_{q}$; and mass fraction and mineral flux boundaries, $\partial \Omega_{c}$ and $\partial \Omega_{j}$. These decomposed boundaries satisfy $\partial \Omega=\overline{\partial \Omega_{u} \cup \partial \Omega_{t}}=\overline{\partial \Omega_{p} \cup \partial \Omega_{q}}=\overline{\partial \Omega_{c} \cup \partial \Omega_{j}}$ and 


$$
\begin{aligned}
& \varnothing=\partial \Omega_{u} \cap \partial \Omega_{t}=\partial \Omega_{p} \cap \partial \Omega_{q}=\partial \Omega_{c} \cap \partial \Omega_{j} \text {. The boundary conditions are given by } \\
& \boldsymbol{u}=\hat{\boldsymbol{u}} \quad \text { on } \quad \partial \Omega_{u} \\
& \boldsymbol{n} \cdot \boldsymbol{\sigma}=\hat{\boldsymbol{t}} \quad \text { on } \quad \partial \Omega_{t} \\
& p=\hat{p} \quad \text { on } \quad \partial \Omega_{p} \\
& \boldsymbol{- n} \cdot \boldsymbol{w}_{l}=\hat{q} \quad \text { on } \quad \partial \Omega_{q} \\
& c=\hat{c} \quad \text { on } \quad \partial \Omega_{c} \\
& \boldsymbol{- n} \cdot\left(c \boldsymbol{w}_{l}+\boldsymbol{j}\right)=\hat{j} \quad \text { on } \quad \partial \Omega_{j},
\end{aligned}
$$

where $\boldsymbol{n}$ is the unit outward normal vector and the hats denote the prescribed boundary values. The initial conditions are given by

$$
\boldsymbol{u}=\boldsymbol{u}_{0}(\boldsymbol{x}), \quad p=p_{0}(\boldsymbol{x}), \quad c=c_{0}(\boldsymbol{x}),
$$

for all position vectors $\boldsymbol{x} \in \Omega$ at time $t=0$. For $s$ and $d$, we consider no flux boundary conditions and zero initial conditions throughout.

\subsection{Time discretization}

We begin the discretization process by approximating the time derivatives in the mass balance equations for liquid, dissolved mineral, and crystals. Given that the chemical, hydrological, and mechanical processes in our problem may involve a variety of time scales, we use an unconditionally stable, first-order backward Euler method. Consider a time increment $\Delta t$ from $t_{n}$ to $t_{n+1}$. We discretize the time derivatives of the primary variables as

$$
\boldsymbol{v}=\dot{\boldsymbol{u}}=\frac{\boldsymbol{u}_{n+1}-\boldsymbol{u}_{n}}{\Delta t}, \quad \dot{p}=\frac{p_{n+1}-p_{n}}{\Delta t}, \quad \dot{c}=\frac{c_{n+1}-c_{n}}{\Delta t}, \quad \dot{S}^{c}=\frac{S_{n+1}^{c}-S_{n}^{c}}{\Delta t},
$$

and the time derivatives of liquid saturation and liquid density as $\dot{S}^{l}=\left(S_{n+1}^{l}-S_{n}^{l}\right) / \Delta t$ and $\dot{\rho}_{l}=\left(\rho_{l, n+1}-\rho_{l, n}\right) / \Delta t$. All other variables are evaluated at time $n+1$. For notational simplicity, hereafter we drop the subscript $n+1$ for quantities at time $t_{n+1}$.

\subsection{Space discretization}

For space discretization we use the finite element method for the deformation and fracture problem (momentum balance and phase-field equations) and the finite volume method for the flow and transport problem (mass balance equations). This combination is motivated by mathematical natures of the two problems. The deformation and fracture problem is described by elliptic equations, so its discretization by the finite element method is an optimal choice. On the other hand, the flow and transport problem involves hyperbolic systems, for which the finite volume method is usually more appropriate than the (continuous) finite element method. This is mainly because finite volume discretization is robust in the presence of sharp gradients in the solution fields as well as locally (element-wise) mass conservative.

We use a single mesh for both finite element and finite volume discretization methods, as shown in Fig. 4. The degrees of freedom for the deformation and fracture unknowns - the displacement vector and the phase field - are located at the element nodes, whereas those for the flow and transport unknowns - the pressure, mass fraction, and crystal saturation - are located at the element center.

To begin finite element discretization of the deformation and fracture problem, we define the spaces of the trial solutions for $\boldsymbol{u}$ and $d$ as

$$
\begin{aligned}
& \mathcal{S}_{u}:=\left\{\boldsymbol{u}: \Omega \rightarrow \mathbb{R}^{\operatorname{dim}} \mid \boldsymbol{u} \in H^{1}, \boldsymbol{u}=\hat{\boldsymbol{u}} \text { on } \partial \Omega_{u}\right\}, \\
& \mathcal{S}_{d}:=\left\{d: \Omega \rightarrow \mathbb{R} \mid d \in H^{1}\right\},
\end{aligned}
$$

where $H^{1}$ is the Sobolev space of order one. Accordingly, the spaces of the weighting functions are defined as

$$
\begin{aligned}
& \mathcal{V}_{u}:=\left\{\boldsymbol{\eta}: \Omega \rightarrow \mathbb{R}^{\mathrm{dim}} \mid \boldsymbol{\eta} \in H^{1}, \boldsymbol{\eta}=\mathbf{0} \text { on } \partial \Omega_{u}\right\}, \\
& \mathcal{V}_{d}:=\left\{\varphi: \Omega \rightarrow \mathbb{R} \mid \varphi \in H^{1}\right\} .
\end{aligned}
$$




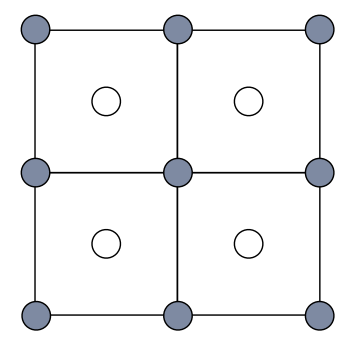

Finite element degrees of freedom

(displacement vector/phase field)

Finite volume degrees of freedom

(pressure/mass fraction/crystal saturation)

Fig. 4. An example mesh illustrating the locations of (linear) finite element and finite volume degrees of freedom.

Through the standard weighted residual procedure, we can readily develop the variational equations of the deformation and fracture problem as

$$
\begin{aligned}
R_{\text {mom }} & :=\int_{\Omega} \nabla^{\mathrm{s}} \boldsymbol{\eta}: \boldsymbol{\sigma}^{\prime} \mathrm{d} V-\int_{\Omega} B \bar{p} \nabla \cdot \boldsymbol{\eta} \mathrm{d} V-\int_{\Omega} \boldsymbol{\eta} \cdot \rho \boldsymbol{g} \mathrm{d} V-\int_{\partial \Omega_{t}} \boldsymbol{\eta} \cdot \hat{\boldsymbol{t}} \mathrm{d} A=0, \\
R_{\text {frac }} & :=\int_{\Omega} \varphi[2(1-d) \mathcal{H}] \mathrm{d} V-\int_{\Omega} \frac{\mathcal{G}_{c}}{l}\left[\varphi+l^{2}(\nabla \varphi \cdot \nabla d)\right] \mathrm{d} V=0 .
\end{aligned}
$$

Here, we have presented the variational equations as residuals to solve these equations via Newton's method later. We then perform finite element discretization of Eqs. (115) and (116), and obtain the discrete residual vectors by assembling element contributions. The contributions of element $e$ are given by

$$
\begin{aligned}
{\left[R_{\mathrm{mom}}\right]^{e} } & :=\int_{\Omega^{e}} \nabla^{s} \eta^{i}: \boldsymbol{\sigma}^{\prime} \mathrm{d} V-\int_{\Omega^{e}} B \bar{p} \nabla \cdot \boldsymbol{\eta}^{i} \mathrm{~d} V-\int_{\Omega^{e}} \eta^{i} \cdot \rho \boldsymbol{g} \mathrm{d} V-\int_{\partial \Omega_{t}^{e}} \eta^{i} \cdot \hat{\boldsymbol{t}} \mathrm{d} A=0, \\
{\left[R_{\mathrm{frac}}\right]^{e} } & :=\int_{\Omega^{e}} \varphi^{i}[2(1-d) \mathcal{H}] \mathrm{d} V-\int_{\Omega^{e}} \frac{\mathcal{G}_{c}}{l}\left[\varphi^{i}+l^{2}\left(\nabla \varphi^{i} \cdot \nabla d\right)\right] \mathrm{d} V=0,
\end{aligned}
$$

where $i$ denotes a shape function index. We use standard linear shape functions in this work.

Next, we discretize the flow and transport problem via the finite volume method, on the same mesh used in the finite element discretization. We integrate the mass balance equations over each element, apply the divergence theorem to the fluid flux terms, and multiply the residuals by the time increment $\Delta t$. Element-wise contributions of the discrete mass residuals are then obtained as

$$
\begin{aligned}
{\left[R_{\text {mass }, 1}\right]^{e}=} & \int_{\Omega^{e}}\left(1-\phi^{s}\right)\left[\rho_{l}\left(S^{l}-S_{n}^{l}\right)+S^{l}\left(\rho_{l}-\rho_{l, n}\right)\right] \mathrm{d} V+\int_{\Omega^{e}} S^{l} \rho_{l} B \nabla \cdot\left(\boldsymbol{u}-\boldsymbol{u}_{n}\right) \mathrm{d} V \\
& -\Delta t \int_{\Omega^{e}} m_{l} \mathrm{~d} V+\Delta t \int_{\partial \Omega^{e}} \boldsymbol{w}_{l} \cdot \boldsymbol{n}^{e} \mathrm{~d} A-\Delta t \int_{\partial \Omega_{q}^{e}} \hat{q} \mathrm{~d} A=0, \\
{\left[R_{\text {mass }, \mathrm{m}}\right]^{e}=} & \int_{\Omega^{e}} \rho^{l}\left(c-c_{n}\right) \mathrm{d} V+\int_{\Omega^{e}} c\left(1-\phi^{s}\right)\left[\rho_{l}\left(S^{l}-S_{n}^{l}\right)+S^{l}\left(\rho_{l}-\rho_{l, n}\right)\right] \mathrm{d} V \\
& +\int_{\Omega^{e}} c S^{l} \rho_{l} B \nabla \cdot\left(\boldsymbol{u}-\boldsymbol{u}_{n}\right) \mathrm{d} V-\Delta t \int_{\Omega^{e}} m_{l} \mathrm{~d} V \\
& +\Delta t \int_{\partial \Omega^{e}}\left(c \boldsymbol{w}_{l}\right) \cdot \boldsymbol{n}^{e} \mathrm{~d} A+\Delta t \int_{\partial \Omega^{e}} \boldsymbol{j} \cdot \boldsymbol{n}^{e} \mathrm{~d} A-\Delta t \int_{\partial \Omega_{j}^{e}} \hat{j} \mathrm{~d} A=0, \\
{\left[R_{\text {mass }, \mathrm{c}}\right]^{e}=} & \int_{\Omega^{e}}\left(1-\phi^{s}\right) \rho_{c}\left(S^{c}-S_{n}^{c}\right) \mathrm{d} V+\int_{\Omega^{e}} S^{c} \rho_{c} B \nabla \cdot\left(\boldsymbol{u}-\boldsymbol{u}_{n}\right) \mathrm{d} V-\Delta t \int_{\Omega^{e}} m_{c} \mathrm{~d} V=0,
\end{aligned}
$$

where $\boldsymbol{n}^{e}$ is the outward normal to the boundary of element $e$. As depicted in Fig. 4, the interpolation functions for the pressure, mass fraction, and crystal saturation fields take a constant value of 1 over element $e$ and 0 at all other elements. Thus the volume integrals can be evaluated much like integrating finite elements with a piecewise constant shape function.

The surface flux integrals are evaluated as a sum of interelement fluxes between element $e$ and its neighboring elements $f$. Let $n_{\text {face }}$ denote the number of faces of element $e$ and $\boldsymbol{n}^{e f}$ denote the outward normal vector at the 
interface $\partial \Omega^{e f}$. The integral of the liquid mass flux is then expressed as

$$
\int_{\partial \Omega^{e}} \boldsymbol{w}_{l} \cdot \boldsymbol{n}^{e} \mathrm{~d} A=\sum_{f=1}^{n_{\text {face }}} \int_{\partial \Omega^{e f}} \boldsymbol{w}_{l} \cdot \boldsymbol{n}^{e f} \mathrm{~d} A=\sum_{f=1}^{n_{\text {face }}}\left(w_{l}\right)^{e f} .
$$

We apply a two-point flux approximation scheme for multiphase flow in porous media as described in [77,78]. Since $\boldsymbol{w}_{l}$ is governed by Darcy's law, $\left(w_{l}\right)^{e f}$ is given by

$$
\left(w_{l}\right)^{e f}=\Gamma^{e f}\left(\Phi^{e}-\Phi^{f}\right) .
$$

Here, $\Phi^{e}=p^{e}+\left(\rho_{l}\right)^{e} g z^{e}$ and $\Phi^{f}=p^{f}+\left(\rho_{l}\right)^{f} g z^{f}$ are the flow potentials at elements $e$ and $f$, respectively, where $g$ denotes the gravitational acceleration and $z^{e}$ and $z^{f}$ denote the elevations of elements $e$ and $f$, respectively. $\Gamma^{e f}$ is the transmissibility at the interface $\partial \Omega^{e f}$, which is multiplicatively decomposed into the geometric transmissibility $T^{e f}$ and the fluid mobility $\left(\lambda_{l}\right)^{e f}$ as

$$
\Gamma^{e f}=T^{e f}\left(\lambda_{l}\right)^{e f} \text {. }
$$

The geometric transmissibility $T^{e f}$ is estimated from the harmonic average of permeabilities of elements $e$ and $f$

$$
T^{e f}=\frac{A^{e f}}{l^{e} / k^{e}+l^{f} / k^{f}},
$$

where $A^{e f}$ is the area of the interface $\partial \Omega^{e f}, l^{e}$ and $l^{f}$ are the distances between the center of the interface and the center of elements $e$ and $f$, respectively, and $k^{e}$ and $k^{f}$ are permeabilities of elements $e$ and $f$, respectively. Note that this term is independent of the flow direction. On the other hand, the fluid mobility $\left(\lambda_{l}\right)^{e f}$ is evaluated in an upstream weighting (upwinding) manner as follows:

$$
\left(\lambda_{l}\right)^{e f}=\left\{\begin{array}{cl}
\rho_{l}^{e} k_{r}^{e} / \mu_{l}^{e} & \text { if } \Phi^{e}>\Phi^{f}, \\
\rho_{l}^{f} k_{r}^{f} / \mu_{l}^{f} & \text { otherwise. }
\end{array}\right.
$$

The remaining flux terms can be computed in a similar manner. For evaluating the surface integral of $c \boldsymbol{w}_{l} \cdot \boldsymbol{n}^{e}, c^{e}$ or $c^{f}$ is multiplied to $\left(\lambda_{l}\right)^{e f}$ in Eq. (126) depending on the flow direction. Modification of the above equations to calculate the interelement fluxes of $\boldsymbol{j}=-\rho_{l} D \nabla c$ is straightforward, and omitted for brevity.

\subsection{Fully discrete form and staggered scheme for phase field}

We now develop the matrix form of the discrete residuals as a linear system that needs to be solved in each Newton iteration. In doing so, we split the overall system into two parts - the phase-field part and the rest - and apply a staggered solution method developed for phase-field modeling of fracture. The primary motivation of using a staggered scheme is its computational robustness: it is far more convergent than a monolithic scheme during the evolution of phase-field fracture. Such robustness is particularly more desirable for phase-field modeling of fracture in strongly coupled multiphysical problems $[37,47]$. The phase-field system, which is uncoupled from other variables, is given by

$$
J_{\mathrm{dd}} \delta d=-R_{\mathrm{frac}}
$$

where $J_{\text {dd }}$ is the Jacobian matrix of $R_{\text {frac }}$ with respect to the phase-field variable $d$, and $\delta$ denotes a Newton increment. Indeed the phase-field system is linear, so $d$ can be updated by solving Eq. (127) once. Next, the rest part - the chemo-hydro-mechanics system - is given by the following block-partitioned system:

$$
\left[\begin{array}{cccc}
J_{\mathrm{uu}} & J_{\mathrm{up}} & J_{\mathrm{uc}} & J_{\mathrm{us}} \\
J_{\mathrm{pu}} & J_{\mathrm{pp}} & J_{\mathrm{pc}} & J_{\mathrm{ps}} \\
J_{\mathrm{cu}} & J_{\mathrm{cp}} & J_{\mathrm{cc}} & J_{\mathrm{cs}} \\
J_{\mathrm{su}} & J_{\mathrm{sp}} & J_{\mathrm{sc}} & J_{\mathrm{ss}}
\end{array}\right]\left\{\begin{array}{l}
\delta \boldsymbol{u} \\
\delta p \\
\delta c \\
\delta s
\end{array}\right\}=-\left\{\begin{array}{l}
R_{\mathrm{mom}} \\
R_{\text {mass }, 1} \\
R_{\text {mass }, \mathrm{d}} \\
R_{\text {mass }, \mathrm{c}}
\end{array}\right\},
$$

where $J_{(\cdot)(\cdot)}$ denotes a sub-matrix in the Jacobian matrix. Specific expressions for the sub-matrices are straightforward to obtain and omitted for brevity. This chemo-hydro-mechanics system is usually nonlinear due to water retention 
characteristics and crystallization kinetics, among other reasons. It is noted that $J_{\mathrm{s}(\cdot)}$ and $J_{(\cdot) \mathrm{s}}$ are nonzero only when the crystals are growing or dissolving in the pores.

Extending the staggered scheme proposed by Miehe et al. [35], we proceed the numerical solution from time $t_{n}$ to $t_{n+1}$ through the following three sub-steps:

1. Determine $\mathcal{H}$ in the phase-field system with the displacement variable $\boldsymbol{u}$ at $t_{n}$.

2. With this $\mathcal{H}$, update the phase-field variable $d$ at $t_{n+1}$, by solving Eq. (127).

3. With the updated $d$, update all other variables $\boldsymbol{u} / p / c / s$ at time $t_{n+1}$, by Newton's method solving Eq. (128) at each iteration.

In the last step, we solve the coupled chemo-hydro-mechanics system in a monolithic manner that updates the primary variables in Eq. (128) together in each iteration. However, other methods that sequentially solve the coupled system (e.g., [79]) can also be used for the same purpose. Such sequentially-implicit methods may be preferred particularly when separate code is available for each type of physical process, e.g., one for the deformation and fracture problem and another for the flow and transport problem. Yet, the analysis of White et al. [80] suggests that, with respect to computational cost per se, a sequential solution approach is suboptimal to a monolithic one employing a decent block-partitioned preconditioner. This motivates the following discussion on a block-partitioned preconditioner for our problem at hand.

\subsection{Block-partitioned preconditioner}

We now seek to design a quality preconditioner for the Jacobian matrix in the coupled chemo-hydro-mechanics system given by Eq. (128). To this end, we extend a block-partitioning strategy originally developed for poromechanics [80-82] to our problem which involves more more types of physical processes. For the purpose of preconditioning, we first consider a subset of the problem whereby crystal kinetics are insignificant (i.e., $J_{\mathrm{s}(\cdot)} \rightarrow 0$ and $J_{(\cdot) \mathrm{s}} \rightarrow 0$ ). In such cases, the Jacobian matrix reduces to

$$
J=\left[\begin{array}{lll}
J_{\mathrm{uu}} & J_{\mathrm{up}} & J_{\mathrm{uc}} \\
J_{\mathrm{pu}} & J_{\mathrm{pp}} & J_{\mathrm{pc}} \\
J_{\mathrm{cu}} & J_{\mathrm{cp}} & J_{\mathrm{cc}}
\end{array}\right],
$$

which is a 3 by 3 block-partitioned matrix. Our particular interest is in developing a block lower-triangular preconditioner of the form

$$
P^{-1}=\left[\begin{array}{ccc}
X & 0 & 0 \\
L_{1} & Y & 0 \\
L_{2} & L_{3} & Z
\end{array}\right],
$$

which, when multiplied to the Jacobian matrix $J$, results in a matrix very close to the block upper-triangular matrix obtained from the LDU factorization of $J$. In other words, we want a block lower-triangular matrix $P^{-1}$ such that

$$
P^{-1} J \approx\left[\begin{array}{ccc}
I & U_{1} & U_{2} \\
0 & I & U_{3} \\
0 & 0 & I
\end{array}\right],
$$

irrespective of $U_{1}, U_{2}$, and $U_{3}$. If this equation is satisfied exactly, the preconditioned Jacobian has a single eigenvalue of 1 and the Jacobian system can be solved by a couple of Krylov iterations. Such an exact preconditioner, however, is generally very expensive to construct.

Therefore, to find an effective yet inexpensive preconditioner, we take the following three-step approach:

1. Find the block preconditioner that exactly satisfies Eq. (131).

2. Approximate dense terms in the exact block preconditioner.

3. Replace inverse operations in the approximated block preconditioner with their own preconditioners.

This approach has been put forward by White and co-workers [80-82] for coupled poromechanics, and it has proven very useful for arriving at a scalable preconditioner. In the following we apply this approach to the coupled chemohydro-mechanics problem of our interest. 
First, we solve for Eq. (131) to find a block lower-triangular matrix $P^{-1}$ that exactly satisfies this equation. We then obtain six equations for the six sub-matrices of $P^{-1}$ as follows:

$$
\begin{aligned}
& X J_{\mathrm{uu}}=I, \\
& L_{1} J_{\mathrm{uu}}+Y J_{\mathrm{pu}}=0, \\
& L_{1} J_{\mathrm{up}}+Y J_{\mathrm{pp}}=I, \\
& L_{2} J_{\mathrm{uu}}+L_{3} J_{\mathrm{pu}}+Z J_{\mathrm{cu}}=0, \\
& L_{2} J_{\mathrm{up}}+L_{3} J_{\mathrm{pp}}+Z J_{\mathrm{cp}}=0, \\
& L_{3} J_{\mathrm{pc}}+Z J_{\mathrm{cc}}=I .
\end{aligned}
$$

The first equation obviously leads to $X=J_{\mathrm{uu}}^{-1}$. From the second and third equations we get

$$
\begin{aligned}
L_{1} & =-Y J_{\mathrm{pu}} J_{\mathrm{uu}}^{-1}, \\
Y & =\left(J_{\mathrm{pp}}-J_{\mathrm{pu}} J_{\mathrm{uu}}^{-1} J_{\mathrm{up}}\right)^{-1} .
\end{aligned}
$$

Here, $J_{\mathrm{pp}}-J_{\mathrm{pu}} J_{\mathrm{uu}}^{-1} J_{\mathrm{up}}$ is the Schur complement of the block $J_{\mathrm{uu}}$ of the upper left 2 by 2 block matrix, which will be denoted by $S_{\mathrm{u}}$ in the following. Solving the rest, we get

$$
\begin{aligned}
L_{2} & =-Z\left[J_{\mathrm{cu}}-\left(J_{\mathrm{cp}}-J_{\mathrm{cu}} J_{\mathrm{uu}}^{-1} J_{\mathrm{up}}\right) S_{\mathrm{u}}^{-1} J_{\mathrm{pu}}\right] J_{\mathrm{uu}}^{-1}, \\
L_{3} & =-Z\left(J_{\mathrm{cp}}-J_{\mathrm{cu}} J_{\mathrm{uu}}^{-1} J_{\mathrm{up}}\right) S_{\mathrm{u}}^{-1} \\
Z & =\left[J_{\mathrm{cc}}-J_{\mathrm{cu}} J_{\mathrm{uu}}^{-1} J_{\mathrm{uc}}-\left(J_{\mathrm{cp}}-J_{\mathrm{cu}} J_{\mathrm{uu}}^{-1} J_{\mathrm{up}}\right) S_{\mathrm{u}}^{-1}\left(J_{\mathrm{pc}}-J_{\mathrm{pu}} J_{\mathrm{uu}}^{-1} J_{\mathrm{uc}}\right)\right]^{-1} .
\end{aligned}
$$

Similar to before, $Z$ appears in $L_{2}$ and $L_{3}$.

Second, we approximate dense terms in the exact preconditioner derived above. Of particular interest is in finding good approximations of those containing $J_{\mathrm{uu}}^{-1}$ multiplied by coupling matrices, e.g., $S_{U}=J_{\mathrm{pp}}-J_{\mathrm{pu}} J_{\mathrm{uu}}^{-1} J_{\mathrm{up}}$. These terms and their inverses appear all blocks of the exact $P^{-1}$ except the $X$ block, but their exact values are overly expensive to be used for a preconditioning purpose. Thus we will approximate these terms, drawing on the "fixedstress" split scheme for poromechanics which has been widely used as a sequential solution method [83-85] and recently rephrased as a block-preconditioning approach [80]. The split scheme goes as follows. If the mean volumetric stress is assumed to be fixed during fluid flow, the divergence of the solid velocity can be related to the change in pore pressure only, i.e.,

$$
\dot{\sigma}_{\mathrm{vol}}=K \nabla \cdot \boldsymbol{v}-B \dot{p}=0 \quad \rightarrow \quad \nabla \cdot v=\frac{B}{K} \dot{p}
$$

where $K$ is the bulk modulus of the solid matrix. Substituting this expression into the mass balance equations for the liquid and dissolved minerals, Eqs. (96) and (99), we get the fixed-stress approximations of these mass balance equations as

$$
\left(1-\phi^{s}\right)\left(\rho_{l} \dot{S}^{l}+S^{l} \dot{\rho}_{l}\right)+S^{l} \rho_{l} \frac{B^{2}}{K} \dot{p}+\nabla \cdot \boldsymbol{w}_{l}=m_{l},
$$

and

$$
\rho^{l} \dot{c}+c\left(1-\phi^{s}\right)\left(\rho_{l} \dot{S}^{l}+S^{l} \dot{\rho}_{l}\right)+c\left(S^{l} \rho_{l} \frac{B^{2}}{K} \dot{p}\right)+\nabla \cdot\left(c \boldsymbol{w}_{l}\right)+\nabla \cdot \boldsymbol{j}=m_{l} .
$$

Observe that the displacement vector $\boldsymbol{u}$ is now eliminated from the original mass balance equations. Discretization of these equations gives sparse approximations of the coupling terms containing $J_{\mathrm{uu}}^{-1}$. For example, the approximated Schur complement, denoted by $\tilde{S}_{U}$, is given by

$$
S_{U} \approx J_{\mathrm{pp}}+S^{l} \rho_{l} \frac{B^{2}}{K} V^{e}:=\tilde{S}_{U},
$$

where $V^{e}$ is the volume of elements assembled in an element-wise manner (corresponds to the mass matrix of the pressure shape functions in finite elements). Here, the original dense term $J_{\mathrm{pu}} J_{\mathrm{uu}}^{-1} J_{\mathrm{up}}$ is approximated by a sparse term (the second term). This approximation reduces to that derived by White et al. [80] in case the material is fully 
saturated $\left(S^{l}=1\right)$ and $\rho_{l}$ is constant. Similarly, we approximate other terms as

$$
\begin{aligned}
& J_{\mathrm{cp}}-J_{\mathrm{cu}} J_{\mathrm{uu}}^{-1} J_{\mathrm{up}} \approx J_{\mathrm{cp}}+c S^{l} \rho_{l} \frac{B^{2}}{K} V^{e}:=\tilde{J}_{\mathrm{cp}}, \\
& J_{\mathrm{pc}}-J_{\mathrm{pu}} J_{\mathrm{uu}}^{-1} J_{\mathrm{uc}} \approx J_{\mathrm{pc}}+S^{l} \frac{\partial \rho_{l}}{\partial c} \frac{B^{2}}{K}\left(p-p_{n}\right) V^{e}:=\tilde{J}_{\mathrm{pc}}, \\
& J_{\mathrm{cc}}-J_{\mathrm{cu}} J_{\mathrm{uu}}^{-1} J_{\mathrm{uc}} \approx J_{\mathrm{cc}}+S^{l} \frac{\partial \rho_{l}}{\partial c} \frac{B^{2}}{K}\left(p-p_{n}\right) V^{e}:=\tilde{J}_{\mathrm{cc}},
\end{aligned}
$$

Accordingly, the $Z$ block is also approximated as

$$
Z \approx\left(\tilde{J}_{\mathrm{cc}}-\tilde{J}_{\mathrm{cp}} \tilde{S}_{\mathrm{u}}^{-1} \tilde{J}_{\mathrm{pc}}\right)^{-1}
$$

This term may be further simplified to $\tilde{J}_{\mathrm{cc}}^{-1}$ or $\left(\tilde{J}_{\mathrm{cc}}-\tilde{J}_{\mathrm{cp}} \operatorname{diag}\left(\tilde{S}_{\mathrm{u}}^{-1}\right) \tilde{J}_{\mathrm{pc}}\right)^{-1}$. We use the former one in this work.

Third, we replace inverse operations in the approximated block preconditioner - which are usually expensive to compute - with their own preconditioners. Specifically,

$$
J_{\mathrm{uu}}^{-1} \approx P_{J_{\mathrm{uu}}}^{-1}, \quad \tilde{S}_{U}^{-1} \approx P_{\tilde{S}_{\mathrm{u}}}^{-1}, \quad \tilde{J}_{\mathrm{cc}}^{-1} \approx P_{\tilde{J}_{\mathrm{cc}}}^{-1},
$$

where $P_{(\cdot)}^{-1}$ is a preconditioner for $(\cdot)^{-1}$. We use algebraic multigrid preconditioners in this work, but a number of other combinations of sub-preconditioners can be efficient as well. Discussions on the choice of sub-preconditioners are presented in [80-82] in the context of poromechanics problems.

Following the three-step procedure described above, we finally arrive at a specific expression for Eq. (130) as

$$
P^{-1}=\left[\begin{array}{rrr}
P_{J_{\mathrm{uu}}}^{-1} & 0 & 0 \\
-P_{\tilde{S}_{\mathrm{u}}}^{-1} J_{\mathrm{pu}} P_{J_{\mathrm{uu}}}^{-1} & P_{\tilde{S}_{\mathrm{u}}}^{-1} & 0 \\
-P_{\tilde{J}_{\mathrm{cc}}}^{-1}\left(J_{\mathrm{cu}}-\tilde{J}_{\mathrm{cp}} P_{\tilde{S}_{\mathrm{u}}}^{-1} \tilde{J}_{\mathrm{pu}}\right) P_{J_{\mathrm{uu}}}^{-1} & -P_{\tilde{J}_{\mathrm{cc}}}^{-1} \tilde{J}_{\mathrm{cp}} P_{\tilde{S}_{\mathrm{u}}}^{-1} & P_{\tilde{J}_{\mathrm{cc}}}^{-1}
\end{array}\right] .
$$

It would be illuminating to demonstrate how this block-preconditioner operates in real problems. Indeed, what is needed by an iterative solution method is the matrix-vector product of the preconditioner and an input vector $\boldsymbol{x}$, say $P^{-1} \boldsymbol{x}=\boldsymbol{y}$. This operation can be written as

$$
\left[\begin{array}{rrr}
P_{J_{\mathrm{uu}}}^{-1} & 0 & 0 \\
-P_{\tilde{S}_{\mathrm{u}}}^{-1} J_{\mathrm{pu}} P_{J_{\mathrm{uu}}}^{-1} & P_{\tilde{S}_{\mathrm{u}}}^{-1} & 0 \\
-P_{\tilde{J}_{\mathrm{cc}}}^{-1}\left(J_{\mathrm{cu}}-\tilde{J}_{\mathrm{cp}} P_{\tilde{S}_{\mathrm{u}}}^{-1} \tilde{J}_{\mathrm{pu}}\right) P_{J_{\mathrm{uu}}}^{-1} & -P_{\tilde{J}_{\mathrm{cc}}}^{-1} \tilde{J}_{\mathrm{cp}} P_{\tilde{S}_{\mathrm{u}}}^{-1} & P_{\tilde{J}_{\mathrm{cc}}}^{-1}
\end{array}\right]\left\{\begin{array}{c}
x_{u} \\
x_{p} \\
x_{c}
\end{array}\right\}=\left\{\begin{array}{c}
y_{u} \\
y_{p} \\
y_{c}
\end{array}\right\} .
$$

The vector $\boldsymbol{y}$ can be efficiently updated via the following three steps:

1. $y_{u}=P_{J_{\mathrm{uu}}}^{-1} x_{u}$

2. $y_{p}=P_{\tilde{S}_{u}}^{-1}\left(x_{p}-J_{\mathrm{pu}} y_{u}\right)$

3. $y_{c}=P_{\tilde{J}_{\mathrm{cc}}}^{-1}\left(x_{c}-J_{\mathrm{cu}} y_{u}-\tilde{J}_{\mathrm{cp}} y_{p}\right)$

Notice that, from the second step, we use the block(s) of $\boldsymbol{y}$ that we updated in previous step(s) $-y_{u}$ in the second step, and $y_{u}$ and $y_{p}$ in the third step. In this sense, this block-preconditioning approach can also be interpreted as a sequential solution method. See White et al. [80] for a thorough discussion on this interpretation. The repeated pattern in this sequence can be further applied to update the additional block related to the crystal kinetics, which we have observed satisfactory results in the numerical examples that follow.

The proposed block-preconditioner has shown decent performance (usually less than 20 Krylov iterations for a tolerance of $10^{-6}$ ) for a number of chemo-hydro-mechanical problems we have tested, and it is believed to be a good choice for other problems sharing a similar mathematical structure. However, much more extensive studies are needed to verify and improve the performance of the current preconditioner-especially with respect to its scalability in large computing platforms, as done by [80-82] for poromechanical problems. To better focus on the scope of the current work, we leave this topic as a future research direction. 


\section{Numerical examples}

In this section, two numerical examples are presented to validate and demonstrate the capability of the developed computational model for simulating complex interactions among unsaturated flow, solid deformation, fracturing, and crystallization in pores. The first example serves as a benchmark problem for verifying the numerical implementation and validating the mathematical model. We simulate a validation problem in unsaturated poromechanics, because a benchmark setting for chemo-hydro-mechanics involving in-pore crystallization is yet to emerge to the best of our knowledge. Subsequently, in the second example we incorporate the flow, transport, and crystallization of minerals and the resulting fracturing process in the host material. We particularly consider the problem of capillary infiltration of a saturated salt solution into a porous medium, which has been commonly used by a number of experimental and numerical studies on crystallization of salts in geomaterials (e.g., [56,86,87]). The main purpose of this example is to showcase the performance of the computational model for simulating the development of fully cracked regions ensuing crystallization in pores.

The numerical examples have been prepared using Geocentric, a massively parallel finite element code for geomechanics. This code is built upon the deal. II finite element library [88,89], p4est mesh handling library [90], and the Trilinos project [91].

\subsection{Drainage of freshwater in a porous column}

Our first example has two purposes: (1) to verify the numerical implementation of combined finite element and finite volume discretization in the current code, and (2) to validate the mathematical model's capability for capturing coupled hydro-mechanical responses that can lead to crystallization of materials. For the latter purpose, we particularly focus on a drainage (saturation decreasing) process whereby supersaturation and crystallization can take place.

To achieve these two purposes simultaneously, we consider a benchmark problem of unsaturated poromechanics that simulates Liakopoulos' drainage experiment of a sand column [92]. This problem has been used by a number of previous studies to validate their own combinations of governing equations, effective stress, water retention, relative permeability, and others (e.g., [93-97]). Here we use this problem in the same context. To verify the implementation, we simulate the drainage experiment with the current code using the finite element method for the solid deformation problem and the finite volume method for the fluid flow problem, and compare the results with those obtained by unsaturated poromechanics code using mixed finite elements for both the solid deformation and fluid flow problems (see $[27,28]$ for details of the mixed finite element discretization). It is noted that, for problems like this one that do not involve a strong advection phenomenon, finite volume and finite element results should converge to the same solution. The numerical results are also compared with the experimental data of Liakopoulos [92] to validate the mathematical model.

Fig. 5 shows a schematic illustration of the problem setup. A $0.1 \mathrm{~m}$ wide and $1.0 \mathrm{~m}$ tall column is prepared such that it is initially under equilibrium with zero pore pressure. The test begins by making the top boundary undrained, which leads to drainage of the pore fluid through the bottom boundary. During the test the bottom boundary is kept zero pressure and fixed, whereas no flux and no lateral displacement are allowed along the side boundaries. Both of the fluid flow and solid deformation in this problem are one-dimensional, and the column is discretized by 80 quadrilateral elements along the height. The same mesh is used for both finite element/finite volume and mixed finite elements simulations. With a uniform time increment $\Delta t=1 \mathrm{~min}$, we simulate the experiment until the drainage time reaches $600 \mathrm{~min}$.

Material parameters are assigned close to the properties of the Del Monte sand tested by Liakopoulos [92]. The material is composed of sand grains and fresh pore water whose intrinsic densities are $\rho_{s}=2700 \mathrm{~kg} / \mathrm{m}^{3}$ and $\rho_{w}=1000 \mathrm{~kg} / \mathrm{m}^{3}$, respectively, with porosity $\phi=1-\phi^{s}=0.2975$. The Biot coefficient $B=1$. As for hydraulic parameters, we use the absolute permeability $k=4.5 \times 10^{-13} \mathrm{~m}^{2}$ (isotropic), the dynamic viscosity $\mu_{l}=10^{-6} \mathrm{kPa}$ $\mathrm{s}$, and the van Genuchten model parameters $S_{1}=0, S_{2}=1, \alpha_{\mathrm{ca}}=15 \mathrm{kPa}$, and $n=5$. Because no mechanical data is available for the tested sand, we assume Young's modulus $E=800 \mathrm{kPa}$ and Poisson's ratio $v=0.25$.

In Fig. 6 we present simulation results obtained by the finite element/finite volume and mixed finite element schemes, along with the experimental data of Liakopoulos [92], in terms of time evolutions of pore pressure variation along the height and fluid velocity at the bottom outlet filter. First, we find that the two numerical schemes yield virtually identical results, thus verify our implementation of combined finite element/finite volume discretization. Second, we see that the mathematical model well reproduces the spatio-temporal evolution of pore pressure measured in the experiment. In Fig. 6(a) the agreement between the simulation and experiments is rather qualitative in the 


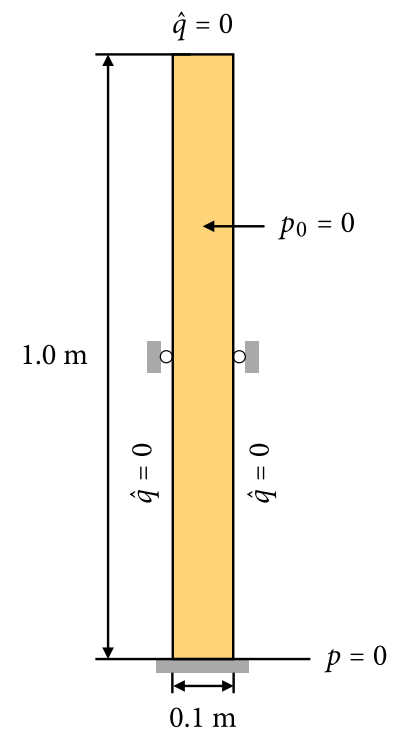

Fig. 5. Schematic illustration of the column drainage example that simulates Liakopoulos' experiment [92]. Initially, the domain is fully saturated with zero pore pressure $\left(p_{0}=0\right)$. Once the simulation begins, the pore fluid is drained through the bottom outlet filter by gravitational force.

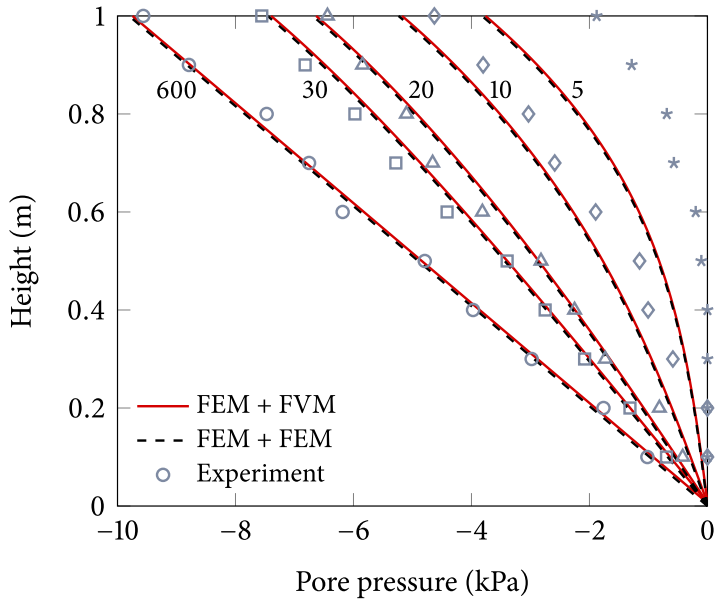

(a)

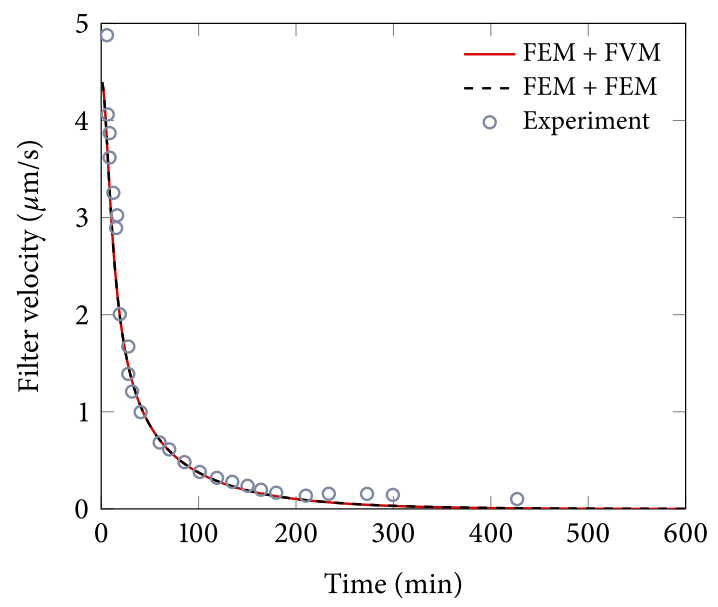

(b)

Fig. 6. Drainage simulation results of the current code using finite elements for the solid deformation problem and finite volume for the fluid flow problem $(\mathrm{FEM}+\mathrm{FVM})$, and an unsaturated poromechanics code using finite elements for both the solid deformation and fluid flow problems (FEM + FEM). The numerical results are compared with experimental data of Liakopoulos [92]. (a) Pore pressure variation along the height (numbers inside the figure denote elapsed time in minutes). (b) Fluid velocity at the bottom outlet filter.

beginning, but it becomes increasingly quantitative as time proceeds. Fig. 6(b) shows that the calculated outlet velocities are in an excellent agreement with the measured data throughout the test. These results demonstrate that, even when the solid behavior is grossly simplified, the computational model can capture salient physics of coupled solid deformation and unsaturated flow in real geomaterials.

\subsection{Capillary infiltration of salt water and crystallization-induced cracking}

We now proceed to the simulation of chemo-hydro-mechanical processes involving crystallization in pores and resulting damage in the solid matrix. Specifically, we simulate a laboratory-scale problem analogous to a typical 


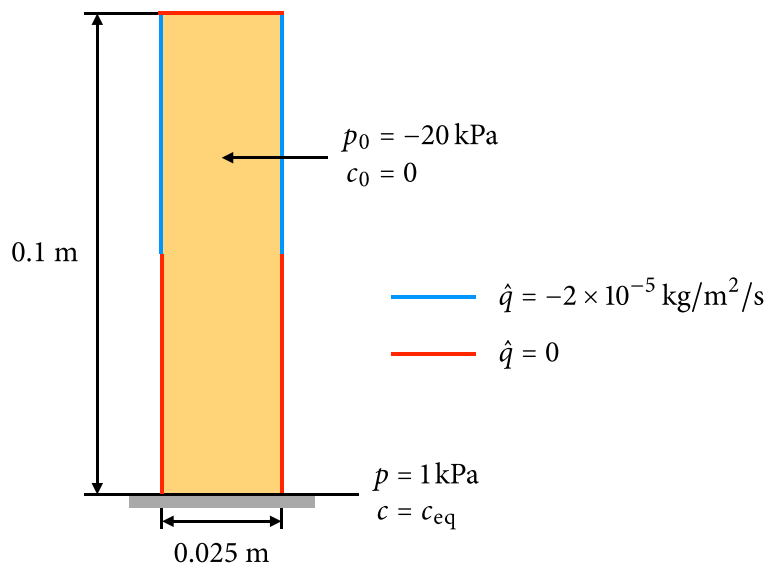

Fig. 7. Schematic illustration of the capillary rise example. The domain is initially filled with freshwater and air such that they manifest a capillary pressure of $20 \mathrm{kPa}$. The simulation begins by prescribing the pressure and mass fraction at the bottom boundary as $1 \mathrm{kPa}$ and $c_{\mathrm{eq}}$, respectively. The lower half of the boundaries are no flux boundaries, whereas the upper half domain is subject to a constant liquid flux of $-2 \times 10^{-5} \mathrm{~kg} / \mathrm{m}^{2} / \mathrm{s}$. Mineral flux $\hat{j}$ is zero throughout the boundaries.

experiment studying growth of salt crystals in geomaterials [86,87], in which a specimen is infiltrated by salt water by capillarity and then damaged by crystallization of salt minerals in pores.

Fig. 7 illustrates the setup of this problem. We consider a porous rock column of width $0.025 \mathrm{~m}$ and height $0.1 \mathrm{~m}$. The column is initially filled with freshwater and air, manifesting a uniform capillary pressure of $20 \mathrm{kPa}$. To emulate capillary rise of a mineral solution, we prescribe the pressure and concentration at the bottom boundary as $1 \mathrm{kPa}$ and $c_{\text {eq }}$ (equilibrium solute mass fraction), respectively. The liquid solution is not allowed to flow outside through the lower half of the lateral boundaries as well as the top boundary, like in the experiment of Noiriel et al. [86]. In contrast, the solution is subject to a constant outward flux boundary condition of $\hat{q}=-2 \times 10^{-5} \mathrm{~kg} / \mathrm{m}^{2} / \mathrm{s}$ in the upper half of the lateral boundaries. Throughout the domain the dissolved minerals are subject to no flux boundary conditions such that the minerals stay in the pores during an outflow of the liquid solution. These boundary conditions are intended to drive supersaturation of the solution - and ultimately crystallization in pores - in the upper half of the specimen. Exploiting the symmetry of the problem, we model the left half of the domain and reflect the result in the post-processing stage. The half domain is discretized by 32768 quadrilateral elements of uniform size (mesh diameter $h=0.025 \mathrm{~cm}$ ), which leads to 198339 degrees of freedom for the five primary variables.

The material is assumed to be similar to the porous rock studied in Rasmussen et al. [98]. We assign the material's properties as follows: the solid density $\rho_{s}=2550 \mathrm{~kg} / \mathrm{m}^{3}$, the initial porosity $\phi_{0}=1-\phi^{s}=0.172$, the Biot coefficient $B=1$, the absolute permeability $k=4.3 \times 10^{-16} \mathrm{~m}^{2}$, and the van Genuchten parameters $S_{1}=0.4, S_{2}=1.0$, $\alpha_{\mathrm{ca}}=50 \mathrm{kPa}$, and $n=1.65$. The linear elasticity parameters of the solid matrix are assumed to be $E=10 \mathrm{GPa}$ and $v=0.25$. The properties of the pore water remain unchanged from the previous example. As for the mineral, we consider salt (sodium chloride), and adopt its parameters mainly from Castellazzi et al. [56]. They are: the mineral density, $\rho_{m}=2160 \mathrm{~kg} / \mathrm{m}^{3}$, the molar volume, $V_{m}=27 \mathrm{~cm}^{3} / \mathrm{mol}$, the equilibrium solute mass fraction, $c_{\mathrm{eq}}=0.264$ $\mathrm{kg} / \mathrm{kg}$, the diffusion coefficient over tortuosity, $D_{m} / \tau=0.5 \times 10^{-9} \mathrm{~m}^{2} / \mathrm{s}$, and the kinetic parameters, $K_{c}=4 \times 10^{-4}$ $\mathrm{kg} / \mathrm{m}^{3} \cdot \mathrm{s}, g_{c}=1.0$, and $U_{\mathrm{thr}}=1.6$. The temperature is set to be $T=293.15 \mathrm{~K}\left(20^{\circ} \mathrm{C}\right)$. Lastly, using Eqs. (90) to (92) with $\beta=0.1453$ [73] and $\sigma_{t}=5 \mathrm{MPa}$, we get the phase-field model parameters as $\mathcal{G}_{c}=49.5 \mathrm{~J} / \mathrm{m}^{2}$ and $l=2.1 \times 10^{-3}$ $\mathrm{m}$. It is noted that the ratio of the length parameter to the mesh diameter $l / h$ is greater than 8 , which has been shown to give sufficient accuracy [36].

Once the simulation begins, the salt water infiltrates into the specimen from the bottom boundary. Fig. 8 shows the evolution of the supersaturation ratio $U$ until $2760 \mathrm{~min}$. At $30 \mathrm{~min}$ and $720 \mathrm{~min}$ the maximum supersaturation ratio equals unity, since no outflow is allowed through the lower half boundaries for both the solution and dissolved minerals. However, when the solution reaches the upper half boundaries, the water in the solution flows outside through the boundaries, whereas the dissolved minerals remain inside the specimen. This type of outflow makes the supersaturation ratio higher than unity. As a result, the contour at $2760 \mathrm{~min}$ show supersaturated zones in which 


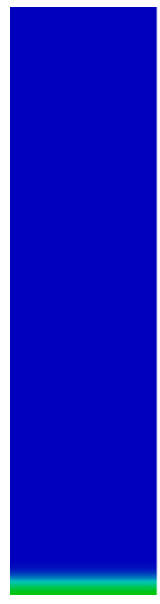

$30.0 \mathrm{~min}$

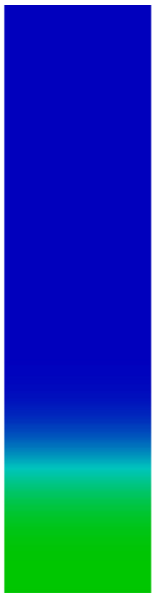

$720.0 \mathrm{~min}$

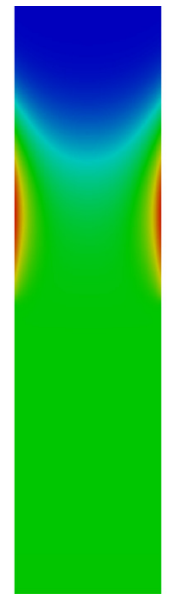

$U$

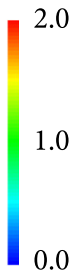

$2760.0 \mathrm{~min}$

Fig. 8. Evolution of the supersaturation ratio $U$ until $2760 \mathrm{~min}$.

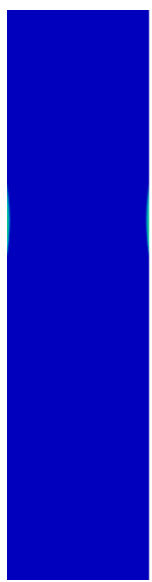

$2760.0 \mathrm{~min}$

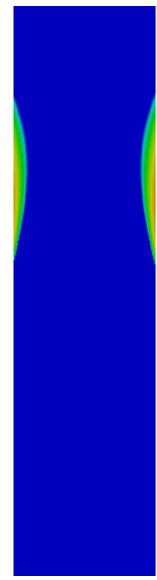

$3426.0 \mathrm{~min}$

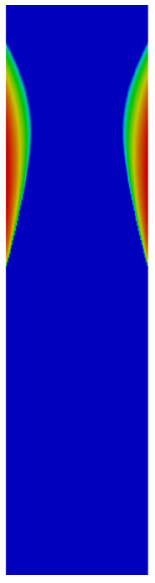

$S^{c}$

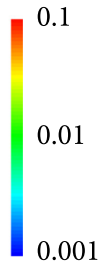

$4092.0 \mathrm{~min}$

Fig. 9. Evolution of the crystal saturation $S^{c}$ from 2760 to $4092 \mathrm{~min}$. Color bar in log scale. (For interpretation of the references to color in this figure legend, the reader is referred to the web version of this article.)

$U>1$. We can also find that the supersaturation ratio exceeds $U_{\mathrm{thr}}=1.6$ in some regions, which means that some minerals have been crystallized.

Figs. 9 and 10 present the crystal saturation ratio, $S^{c}$, and the crystallization pressure, $p_{\mathrm{cr}}$, at three time instances from 2760 to $4092 \mathrm{~min}$. Fig. 9 shows that during this time crystals grow continuously at the upper half of the lateral boundaries, which may be viewed as salt efflorescence. We can also see from Fig. 10 that this crystal growth leads to an increase in the crystallization pressure. Recall that the crystallization pressure enters the mean pore pressure in effective stress. As such, this increase in the crystallization pressure can give rise to tensile effective stress which drives the evolution of the phase-field variable.

In Fig. 11 we plot the evolution of the phase-field variable $d$ from 4092 min, with a uniform time interval of $0.1 \mathrm{~min}$. Until $4092.1 \mathrm{~min}$ the phase-field value does not reach 1.0, so the material may be regarded as partially damaged by microcracks. After $0.1 \mathrm{~min}$, the phase-field value evolves to 1 at some locations, which means that the material is fully cracked therein. The cracked zones enlarge further in the next $0.1 \mathrm{~min}$. It is noted that the shape of the cracked regions at 4092.3 min resembles characteristic cave-like damage zones in salt weathering. To the best of 


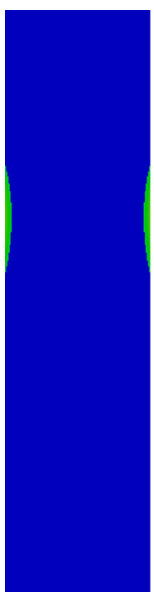

$2760.0 \mathrm{~min}$

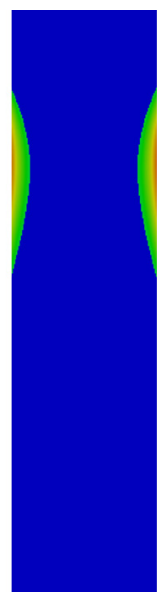

$3426.0 \mathrm{~min}$

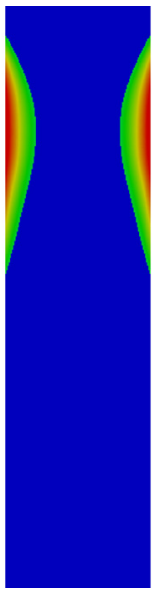

$p_{\text {cr }}$

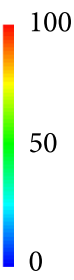

$4092.0 \mathrm{~min}$

Fig. 10. Evolution of the crystallization pressure $p_{\text {cr }}$ from 2760 to $4092 \mathrm{~min}$. Color bar in MPa. (For interpretation of the references to color in this figure legend, the reader is referred to the web version of this article.)

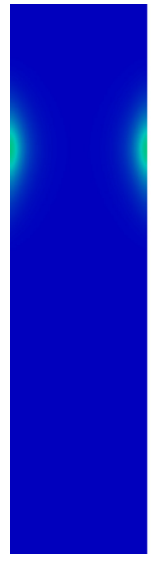

$4092.0 \mathrm{~min}$

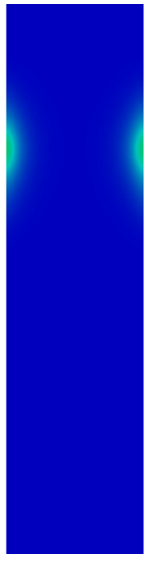

$4092.1 \mathrm{~min}$

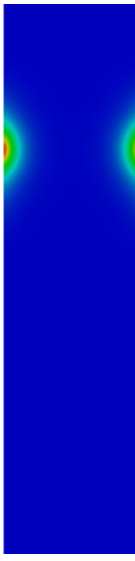

$4092.2 \mathrm{~min}$

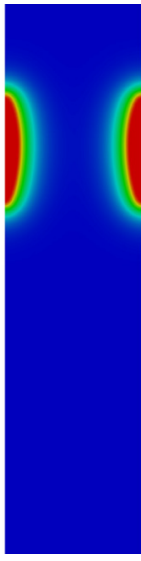

$4092.3 \mathrm{~min}$ $d$

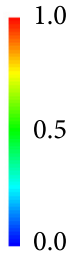

0.0

Fig. 11. Evolution of the phase-field variable $d$ from 4092.0 to $4092.3 \mathrm{~min}$.

our knowledge, this is the first numerical simulation of the evolution of damage and fully-cracked zones by in-pore crystallization of minerals.

The simulation is terminated at this point, because many assumptions in the constitutive models break down upon fracturing of the material. For example, the crystallization pressure equations in the literature have been proposed for a crystal confined in small pore space such that a solution film exists between the crystal and the solid matrix. Therefore, when cracks develop significantly as $4092.3 \mathrm{~min}$ in Fig. 11, these equations might become invalid or the crystallization pressure might disappear. Also, we have assumed that the kinetic parameter $K_{c}$ is constant, but it is expected to evolve by the fracturing process. Note, however, that all these limitations stem from the lack of constitutive relations for crystals within fractures, not from the modeling framework. Once constitutive models that address the aforementioned issues become available, they can be cast into the current modeling framework.

Lastly, we would like to discuss computational aspects of the problem. As shown in Fig. 11, the phase-field variable has evolved from partial damage to full cracks dramatically within the last $0.2 \mathrm{~min}$. To capture such an accelerated cracking process, we have used an adaptive time stepping algorithm proposed by Borden et al. [41]. As a result, during the cracking process the time increments have been reduced to very small numbers (orders of magnitude smaller than a minute), giving rise to undrained deformations in which the relative flow of the pore fluid is negligible. This aspect 
further justifies our choice of finite volume discretization for the flow problem, because mixed finite elements for coupled poromechanics are subject to an inf-sup stability condition in undrained conditions [99-104]. We also would like to mention that solving a large number of steps in the crack development stage has been made affordable thanks to the block-partitioned preconditioner described in the previous section. This preconditioner has allowed us to solve each linear system via a couple of dozens Krylov iterations until large cracked zones emerge.

\section{Closure}

This paper has presented a theoretical and computational framework for modeling cracking and damage in porous materials from in-pore crystallization of minerals. The framework combines a chemo-hydro-mechanics approach with a phase-field description of fracture. Particular contributions of this work include: (1) derivation of a thermodynamically consistent effective stress tensor in porous materials containing growing crystals, (2) identification of state variables that must be linked via constitutive laws, and (3) a block-preconditioned iterative solver that facilitates numerical solution of the fully coupled chemo-hydro-mechanics equations. The computational model has been demonstrated to be capable of simulating the onset and evolution of cracking and damage from in-pore crystallization of minerals.

In this work we have proposed a general modeling framework and then constructed its particular class by employing relatively simple constitutive models of crystallization kinetics and solid deformation. We would like to note that use of more advanced constitutive models for these physical processes may lead to other classes that have better predictive capabilities. Particularly, if quantifiable, pore-scale characteristics need to be incorporated into the crystallization kinetics model. An important example is the pore size distribution because crystallization in real geomaterials begins from smaller pores. The orientation of the mineral crystal may also exert control on this problem [105]. Such multiphysical processes at the pore scale may be accommodated by microscale simulations in conjunction with multiscale modeling methods [106-111]. As for the solid model, incorporating pressure-dependent plasticity will allow us to accommodate the effect of confining pressure on fracturing [54], which is important for deep subsurface problems. Beyond constitutive models, the temperature field must be augmented to the formulation when heat flow is significant. For problems involving complex drying phenomena, one needs to introduce additional phases for vapors and models of moisture transport [112-114]. The framework developed in this work is amenable to incorporating these more complicated physical processes.

\section{Acknowledgments}

The authors are grateful to Prof. Peter B. Kelemen and Prof. Marc W. Spiegelman for the enlightening discussions that motivated this work and provided important physical insights into the process of crystallization in pores. They also thank Dr. Joshua A. White for his valuable assistance in the implementation of the finite volume method.

Financial support for the first author has been provided by the Integrated Earth Systems program of the National Science Foundation under Award Number EAR-1516300 for the development of chemo-hydro-mechanics theory of the crystallization problem, as well as the Mechanics of Materials and Structures program of the National Science Foundation under Award Number CMMI-1462760 for the derivation, implementation, verification, and validation of the phase-field model and the computational chemo-hydro-mechanics model. The work of the second author has been supported by the Earth Materials and Processes program of the US Army Research Office under Award Numbers W911NF-15-1-0442 and W911NF-15-1-0581, and the Dynamic Materials and Interactions program of the Air Force Office of Scientific Research under Award Number FA9550-17-1-0169. Support from these sponsors is greatly appreciated.

The views and conclusions contained in this document are those of the authors, and should not be interpreted as representing the official policies, either expressed or implied, of the sponsors, including the Army Research Laboratory or the U.S. Government. The U.S. Government is authorized to reproduce and distribute reprints for Government purposes notwithstanding any copyright notation herein.

\section{References}

[1] Conservation of Historic Stone Buildings and Monuments, The National Academies Press, Washington, D.C., 1982.

[2] N. Tsui, R.J. Flatt, G.W. Scherer, Crystallization damage by sodium sulfate, J. Cultural Heritage 4 (2) (2003) 109-115. 
[3] M. Angeli, J.P. Bigas, D. Benavente, B. Menéndez, R. Hébert, C. David, Salt crystallization in pores: Quantification and estimation of damage, Environ. Geol. 52 (2) (2007) 187-195.

[4] R.M. Espinosa-Marzal, G.W. Scherer, Mechanisms of damage by salt, Geologic. Soc. Lond. Spec. Publ. 331 (1) (2010) 61-77.

[5] M. Steiger, A.E. Charola, K. Sterflinger, Weathering and deterioration, in: Stone in Architecture, Springer, 2014, pp. 225-316.

[6] R.J. Flatt, F. Caruso, A.M.A. Sanchez, G.W. Scherer, Chemo-mechanics of salt damage in stone, Nat. Commun. 5 (2014) 4823.

[7] I. Sass, U. Burbaum, Damage to the historic town of Staufen (Germany) caused by geothermal drillings through anhydrite-bearing formations, Acta Carsologica 39 (2) (2010) 233-245.

[8] K. Serafeimidis, G. Anagnostou, On the time-development of sulphate hydration in anhydritic swelling rocks, Rock Mech. Rock Eng. 46 (3) (2013) 619-634.

[9] E.E. Alonso, A. Ramon, Massive sulfate attack to cement-treated railway embankments, Géotechnique 63 (10) (2013) 857-870.

[10] P.B. Kelemen, J. Matter, In situ carbonation of peridotite for $\mathrm{CO}_{2}$ storage, Proc. Natl. Acad. Sci. USA 105 (45) (2008) $17295-17300$.

[11] G.W. Scherer, Crystallization in pores, Cement Concrete Res. 29 (8) (1999) 1347-1358.

[12] G.W. Scherer, Stress from crystallization of salt, Cement Concrete Res. 34 (9) (2004) 1613-1624.

[13] C.W. Correns, W. Steinborn, Experimente zur Messung und Erklärung der sogenannten Kristallisationskraft, Z. Kristallogr. 101 (1939) $117-133$.

[14] C.W. Correns, Growth and dissolution of crystals under linear pressure, Discuss. Faraday Soc. 5 (1949) 267-271.

[15] M. Steiger, Crystal growth in porous materials-I: The crystallization pressure of large crystals, J. Cryst. Growth 282 (3-4) (2005) 455-469.

[16] M. Steiger, Crystal growth in porous materials-II: Influence of crystal size on the crystallization pressure, J. Cryst. Growth 282 (3-4) (2005) 470-481.

[17] R.J. Flatt, G.W. Scherer, Thermodynamics of crystallization stresses in DEF, Cement Concrete Res. 38 (3) (2008) 325-336.

[18] P.B. Kelemen, G. Hirth, Reaction-driven cracking during retrograde metamorphism: Olivine hydration and carbonation, Earth Planet. Sci. Lett. 345-348 (2012) 81-89.

[19] J. Desarnaud, D. Bonn, N. Shahidzadeh, The pressure induced by salt crystallization in confinement, Sci. Rep. 6 (1) (2016) 30856.

[20] O. Coussy, Deformation and stress from in-pore drying-induced crystallization of salt, J. Mech. Phys. Solids 54 (8) (2006) $1517-1547$.

[21] M. Koniorczyk, D. Gawin, Modelling of salt crystallization in building materials with microstructure - Poromechanical approach, Constr. Build. Mater. 36 (2012) 860-873.

[22] H. Derluyn, P. Moonen, J. Carmeliet, Deformation and damage due to drying-induced salt crystallization in porous limestone, J. Mech. Phys. Solids 63 (1) (2014) 242-255.

[23] R.I. Borja, Cam-Clay plasticity. Part V: A mathematical framework for three-phase deformation and strain localization analyses of partially saturated porous media, Comput. Methods Appl. Mech. Engrg. 193 (48-51) (2004) 5301-5338.

[24] R.I. Borja, On the mechanical energy and effective stress in saturated and unsaturated porous continua, Int. J. Solids Struct. 43 (6) (2006) $1764-1786$.

[25] R.I. Borja, A. Koliji, On the effective stress in unsaturated porous continua with double porosity, J. Mech. Phys. Solids 57 (8) (2009) $1182-1193$.

[26] X. Song, R.I. Borja, Mathematical framework for unsaturated flow in the finite deformation range, Internat. J. Numer. Methods Engrg. 97 (2014) 658-682.

[27] J. Choo, J.A. White, R.I. Borja, Hydromechanical modeling of unsaturated flow in double porosity media, Int. J. Geomech. 16 (6) (2016) D4016002.

[28] J. Choo, Hydromechanical modeling framework for multiscale porous materials, Stanford University, 2016.

[29] R.I. Borja, J. Choo, Cam-Clay plasticity, Part VIII: A constitutive framework for porous materials with evolving internal structure, Comput. Methods Appl. Mech. Engrg. 309 (2016) 653-679.

[30] R.I. Borja, J.A. White, Continuum deformation and stability analyses of a steep hillside slope under rainfall infiltration, Acta Geotech. 5 (1) (2010) $1-14$.

[31] R.I. Borja, J. Choo, J.A. White, Rock moisture dynamics, preferential flow, and the stability of hillside slopes, in: P. Gardoni, J.M. LaFave (Eds.), Multi-hazard Approaches to Civil Infrastructure Engineering, Springer, 2016, pp. 443-464.

[32] B. Bourdin, G.A. Francfort, J.J. Marigo, The variational approach to fracture, J. Elasticity 91 (2008) 5-148.

[33] H. Amor, J.J. Marigo, C. Maurini, Regularized formulation of the variational brittle fracture with unilateral contact: Numerical experiments, J. Mech. Phys. Solids 57 (8) (2009) 1209-1229.

[34] C. Miehe, F. Welschinger, M. Hofacker, Thermodynamically consistent phase-field models of fracture: Variational principles and multi-field FE implementations, Internat. J. Numer. Methods Engrg. 82 (2010) 1273-1311.

[35] C. Miehe, M. Hofacker, F. Welschinger, A phase field model for rate-independent crack propagation: Robust algorithmic implementation based on operator splits, Comput. Methods Appl. Mech. Engrg. 199 (45-48) (2010) 2765-2778.

[36] M.J. Borden, C.V. Verhoosel, M.A. Scott, T.J.R. Hughes, C.M. Landis, A phase-field description of dynamic brittle fracture, Comput. Methods Appl. Mech. Engrg. 217-220 (2012) 77-95.

[37] Z.A. Wilson, M.J. Borden, C.M. Landis, A phase-field model for fracture in piezoelectric ceramics, Int. J. Fract. 183 (2) (2013) $135-153$.

[38] M.J. Borden, T.J.R. Hughes, C.M. Landis, C.V. Verhoosel, A higher-order phase-field model for brittle fracture: Formulation and analysis within the isogeometric analysis framework, Comput. Methods Appl. Mech. Engrg. 273 (2014) 100-118.

[39] A. Mikelić, M. Wheeler, T. Wick, A phase-field method for propagating fluid-filled fractures coupled to a surrounding porous medium, Multiscale Model. Simul. 13 (1) (2015) 367-398.

[40] C. Miehe, S. Mauthe, Phase field modeling of fracture in multi-physics problems. Part III. Crack driving forces in hydro-poro-elasticity and hydraulic fracturing of fluid-saturated porous media, Comput. Methods Appl. Mech. Engrg. 304 (2015) 619-655. 
[41] M.J. Borden, T.J.R. Hughes, C.M. Landis, A. Anvari, I.J. Lee, A phase-field formulation for fracture in ductile materials: Finite deformation balance law derivation, plastic degradation, and stress triaxiality effects, Comput. Methods Appl. Mech. Engrg. 312 (2016) 130-166.

[42] Z.A. Wilson, C.M. Landis, Phase-field modeling of hydraulic fracture, J. Mech. Phys. Solids 96 (2016) 264-290.

[43] S. Lee, A. Mikelić, M.F. Wheeler, T. Wick, Phase-field modeling of proppant-filled fractures in a poroelastic medium, Comput. Methods Appl. Mech. Engrg. 312 (2016) 509-541.

[44] S. Lee, J.E. Reber, N.W. Hayman, M.F. Wheeler, Investigation of wing crack formation with a combined phase-field and experimental approach, Geophys. Res. Lett. 43 (15) (2016) 7946-7952.

[45] S. Lee, M.F. Wheeler, T. Wick, Pressure and fluid-driven fracture propagation in porous media using an adaptive finite element phase field model, Comput. Methods Appl. Mech. Engrg. 305 (2016) 111-132.

[46] L. De Lorenzis, A. McBride, B. Reddy, Phase-field modelling of fracture in single crystal plasticity, GAMM-Mitt. 39 (1) (2016) 7-34.

[47] X. Zhang, A. Krischok, C. Linder, A variational framework to model diffusion induced large plastic deformation and phase field fracture during initial two-phase lithiation of silicon electrodes, Comput. Methods Appl. Mech. Engrg. 312 (2016) 51-77.

[48] S. Lee, M.F. Wheeler, T. Wick, Iterative coupling of flow, geomechanics and adaptive phase-field fracture including level-set crack width approaches, J. Comput. Appl. Math. 314 (2017) 40-60.

[49] S. Mauthe, C. Miehe, Hydraulic fracture in poro-hydro-elastic media, Mech. Res. Commun. 80 (2017) 69-83.

[50] W. Ehlers, C. Luo, A phase-field approach embedded in the Theory of Porous Media for the description of dynamic hydraulic fracturing, Comput. Methods Appl. Mech. Engrg. 315 (2017) 348-368.

[51] D. Santillán, R. Juanes, L. Cueto-Felgueroso, Phase-field model of fluid-driven fracture in elastic media: immersed-fracture formulation and validation with analytical solutions, J. Geophys. Res.: Solid Earth (2017) 1-25.

[52] S. Na, W.C. Sun, H. Yoon, M. Ingraham, Effects of elastic heterogeneity on the fracture pattern and macroscopic effective toughness of Mancos Shale in Brazilian tests, J. Geophys. Res.: Solid Earth 122 (8) (2017) 6202-6230.

[53] T. Cajuhi, L. Sanavia, L. De Lorenzis, Phase-field modeling of fracture in variably saturated porous media, Comput. Mech. (2017). http://dx.doi.org/10.1007/s00466-017-1459-3.

[54] J. Choo, W. Sun, Coupled phase-field and plasticity modeling of geological materials: From brittle fracture to ductile flow, Comput. Methods Appl. Mech. Engrg. 330 (2018) 1-32.

[55] R. de Borst, C.V. Verhoosel, Gradient damage vs phase-field approaches for fracture: Similarities and differences, Comput. Methods Appl. Mech. Engrg. 312 (2016) 78-94.

[56] G. Castellazzi, C. Colla, S. De Miranda, G. Formica, E. Gabrielli, L. Molari, F. Ubertini, A coupled multiphase model for hygrothermal analysis of masonry structures and prediction of stress induced by salt crystallization, Constr. Build. Mater. 41 (2013) 717-731.

[57] S. Na, W. Sun, Computational thermo-hydro-mechanics for multiphase freezing and thawing porous media in the finite deformation range, Comput. Methods Appl. Mech. Engrg. 318 (2017) 667-700.

[58] K. Terzaghi, Theoretical Soil Mechanics, John Wiley \& Sons, 1943.

[59] J. Choo, Y.-H. Jung, C.-K. Chung, Effect of directional stress history on anisotropy of initial stiffness of cohesive soils measured by bender element tests, Soils Found. 51 (4) (2011) 737-747.

[60] Y.-H. Jung, J. Choo, W. Cho, C.-K. Chung, Patterns of nonlinear shear stiffness degradation of reconstituted clay with different stress histories, Marine Geores. Geotechnol. 31 (4) (2013) 309-331.

[61] J. Choo, Y.-H. Jung, W. Cho, C.-K. Chung, Effect of pre-shear stress path on nonlinear shear stiffness degradation of cohesive soils, Geotech. Test. J. 36 (2) (2013) 198-205.

[62] R.J. Flatt, M. Steiger, G.W. Scherer, A commented translation of the paper by C.W. Correns and W. Steinborn on crystallization pressure, Environ. Geol. 52 (2007) 187-203.

[63] M.Th. van Genuchten, A closed-form equation for predicting the hydraulic conductivity of unsaturated soils, Soil Sci. Am. J. 44 (5) (1980) 892-898.

[64] R.M. Espinosa-Marzal, G.W. Scherer, Impact of in-pore salt crystallization on transport properties, Environ. Earth Sci. 69 (8) (2013) $2657-2669$.

[65] R.M. Espinosa-Marzal, L. Franke, G. Deckelmann, Phase changes of salts in porous materials: Crystallization, hydration and deliquescence, Constr. Build. Mater. 22 (2007) 1758-1773.

[66] M.E. Gurtin, Generalized Cahn-Hilliard equations based on a microforce balance, Physica D 92 (1996) 178-192.

[67] M.E. Gurtin, On the plasticity of single crystals: Free energy, microforces, plastic strain gradients, J. Mech. Phys. Solids 48 (2000) $989-1036$.

[68] D.L. Henann, K. Kamrin, Continuum thermomechanics of the nonlocal granular rheology, Int. J. Plast. 60 (2014) 145-162.

[69] A. Griffith, The phenomena of rupture and flow in solids, Phil. Trans. Ser. A 221 (1921) 163-198.

[70] K. Wang, W. Sun, A unified variational eigen-erosion framework for interacting brittle fractures and compaction bands in fluid-infiltrating porous media, Comput. Methods Appl. Mech. Engrg. 318 (2017) 1-32.

[71] X. Zhang, C. Vignes, S.W. Sloan, D. Sheng, Numerical evaluation of the phase-field model for brittle fracture with emphasis on the length scale, Comput. Mech. 59 (5) (2017) 737-752.

[72] J.A. Harison, B.O. Hardin, K. Mahboub, Fracture toughness of compacted cohesive soils using ring test, J. Geotech. Eng. 120 (5) (1994) 872-891.

[73] Z.X. Zhang, An empirical relation between mode-I fracture toughness and the tensile strength of rock, Int. J. Rock Mech. Mining Sci. 39 (2002) 401-406.

[74] J. Wang, J. Zhu, C. Chiu, H. Zhang, Experimental study on fracture toughness and tensile strength of a clay, Eng. Geol. 94 (1-2) (2007) $65-75$. 
[75] M. Lakshmikantha, P. Prat, A. Ledesma, Discussion on "Experimental study on fracture toughness and tensile strength of a clay [Engineering Geology 94 (2007) 64-75], Eng. Geol. 101 (2008) 295-296.

[76] R.I. Borja, J.A. White, Conservation laws for coupled hydromechanical processes in unsaturated porous media: Theory and implementation, in: L. Laloui (Ed.), Mechanics of Unsaturated Geomaterials, John Wiley \& Sons, 2010, pp. 185-208.

[77] K. Aziz, L. Durlofsky, H.A. Tchelepi, Notes on Reservoir Simulation, Stanford Unviersity Lecture Notes, 2014.

[78] B. Jha, R. Juanes, Coupled multiphase flow and poromechanics: A computational model of pore pressure effects on fault slip and earthquake triggering, Water Resour. Res. 50 (2014).

[79] J. Kim, E. Sonnenthal, J. Rutqvist, A sequential implicit algorithm of chemo-thermo-poro-mechanics for fractured geothermal reservoirs, Comput. Geosci. 76 (2015) 59-71.

[80] J.A. White, N. Castelletto, H.A. Tchelepi, Block-partitioned solvers for coupled poromechanics: A unified framework, Comput. Methods Appl. Mech. Engrg. 303 (2016) 55-74.

[81] J.A. White, R.I. Borja, Block-preconditioned Newton-Krylov solvers for fully coupled flow and geomechanics, Comput. Geosci. 15 (4) (2011) 647-659.

[82] N. Castelletto, J.A. White, M. Ferronato, Scalable algorithms for three-field mixed finite element coupled poromechanics, J. Comput. Phys. 327 (2016) 894-918.

[83] J. Kim, H.A. Tchelepi, R. Juanes, Stability, accuracy and efficiency of sequential methods for coupled flow and geomechanics, SPE J. 16 (2) (2011) 249-262.

[84] J. Kim, H.A. Tchelepi, R. Juanes, Stability and convergence of sequential methods for coupled flow and geomechanics: Fixed stress and fixed-strain splits, Comput. Methods Appl. Mech. Engrg. 200 (2011) 1591-1606.

[85] A. Mikelić, M.F. Wheeler, Convergence of iterative coupling for coupled flow and geomechanics, Comput. Geosci. 17 (2013) $455-461$.

[86] C. Noiriel, F. Renard, M.L. Doan, J.P. Gratier, Intense fracturing and fracture sealing induced by mineral growth in porous rocks, Chem. Geol. 269 (3-4) (2010) 197-209.

[87] S. Dai, H. Shin, J.C. Santamarina, Formation and development of salt crusts on soil surfaces, Acta Geotech. 11 (5) (2016) $1103-1109$.

[88] W. Bangerth, R. Hartmann, G. Kanschat, (2007) deal . II-A general-purpose object-oriented finite element library, 33 (4) 1-27.

[89] D. Arndt, W. Bangerth, D. Davydov, T. Heister, L. Heltai, M. Kronbichler, M. Maier, J.-P. Pelteret, B. Turcksin, D. Wells, The deal . II library, version 8.5, J. Numer. Math. 25 (3) (2017) 137-146.

[90] C. Burstedde, L.C. Wilcox, O. Ghattas, p4est: Scalable algorithms for parallel adaptive mesh refinement on forests of octrees, SIAM J. Sci. Comput. 33 (3) (2011) 1103-1133.

[91] M.A. Heroux, J.M. Willenbring, A new overview of the Trilinos project, Sci. Program. 20 (2) (2012) 83-88.

[92] A.C. Liakopoulos, Transient Flow Through Unsaturated Porous Media, University of California, Berkeley, 1964.

[93] B.A. Schrefler, Z. Xiaoyong, A fully coupled model for water flow and airflow in deformable porous media, Water Resour. Res. 29 (1) (1993) $155-167$.

[94] G. Klubertanz, L. Laloui, L. Vulliet, (1997) Numerical modeling of unsaturated porous media as a two and three phase medium: A comparison, in: Proc. 9th Int. Conf. On Comp. Meth. and Advances in Geomech., IACMAG Vol. 2, pp. 1159-1164.

[95] B.A. Schrefler, R. Scotta, A fully coupled dynamic model for two-phase fluid flow in deformable porous media, Comput. Methods Appl. Mech. Engrg. 190 (24-25) (2001) 3223-3246.

[96] W. Ehlers, T. Graf, M. Ammann, Deformation and localization analysis of partially saturated soil, Comput. Methods Appl. Mech. Engrg. 193 (27-29) (2004) 2885-2910.

[97] E. Liu, H.S. Yu, G. Deng, J. Zhang, S. He, Numerical analysis of seepage-deformation in unsaturated soils, Acta Geotech. 9 (6) (2014) $1045-1058$

[98] T.C. Rasmussen, D.D. Evans, P.J. Sheets, J.H. Blandford, Permeability of Apace Leap Tuff: Borehole and core measurements using water and air, Water Resour. Res. 29 (7) (1993) 1997-2006.

[99] J.A. White, R.I. Borja, Stabilized low-order finite elements for coupled solid-deformation/fluid-diffusion and their application to fault zone transients, Comput. Methods Appl. Mech. Engrg. 197 (49-50) (2008) 4353-4366.

[100] W. Sun, J.T. Ostien, A.G. Salinger, A stabilized assumed deformation gradient finite element formulation for strongly coupled poromechanical simulations at finite strain, Int. J. Numer. Anal. Methods Geomech. 37 (16) (2013) 2755-2788.

[101] J. Choo, R.I. Borja, Stabilized mixed finite elements for deformable porous media with double porosity, Comput. Methods Appl. Mech. Engrg. 293 (2015) 131-154.

[102] W. Sun, A stabilized finite element formulation for monolithic thermo-hydro-mechanical simulations at finite strain, Internat. J. Numer. Methods Engrg. 103 (11) (2015) 798-839.

[103] A. Krischok, C. Linder, On the enhancement of low-order mixed finite element methods for the large deformation analysis of diffusion in solids, Internat. J. Numer. Methods Engrg. 106 (4) (2016) 278-297.

[104] B. Dortdivanlioglu, A. Krischok, L. Beirão da Veiga, C. Linder, Mixed isogeometric analysis of strongly coupled diffusion in porous materials, Internat. J. Numer. Methods Engrg., http://dx.doi.org/10.1002/nme.5731.

[105] B. Lecampion, Stress-induced crystal preferred orientation in the poromechanics of in-pore crystallization, J. Mech. Phys. Solids 58 (10) (2010) 1701-1715.

[106] J. White, R. Borja, J. Fredrich, Calculating the effective permeability of sandstone with multiscale lattice Boltzmann/finite element simulations, Acta Geotech. 1 (2006) 195-209.

[107] W. Sun, J.E. Andrade, J.W. Rudnicki, Multiscale method for characterization of porous microstructures and their impact on macroscopic effective permeability, Internat. J. Numer. Methods Engrg. 88 (12) (2011) 1260-1279. 
[108] J. Choo, Y.J. Kim, J. Lee, T.S. Yun, J. Lee, Y.S. Kim, Stress-induced evolution of anisotropic thermal conductivity of dry granular materials, Acta Geotech. 8 (1) (2013) 91-106.

[109] D.H. Kang, J. Choo, T.S. Yun, Evolution of pore characteristics in the 3D numerical direct shear test, Comput. Geotech. 49 (2013) 53-61.

[110] W. Sun, M.R. Kuhn, J.W. Rudnicki, A multiscale DEM-LBM analysis on permeability evolutions inside a dilatant shear band, Acta Geotech. (2013) $1-16$.

[111] W. Sun, Z. Cai, J. Choo, Mixed Arlequin method for multiscale poromechanics problems, Internat. J. Numer. Methods Engrg. 111 (7) (2017) $624-659$.

[112] G.W. Scherer, Theory of drying, J. Amer. Ceramic Soc. 73 (1) (1990) 3-14.

[113] S.J. Kowalski, A. Rybicki, Drying stress formation induced by inhomogeneous moisture and temperature distribution, Transp. Porous Media 24 (1996) 239-248.

[114] S.J. Kowalski, Thermomechanics of Drying Processes, Springer, 2003. 